\title{
PLETHORA OF PLANTS - COLLECTIONS OF THE BOTANICAL GARDEN, FACULTY OF SCIENCE, UNIVERSITY OF ZAGREB (3): IRIS (IRIDACEAE) COLLECTION
}

\author{
SANJa KovačIĆ \\ Botanical Garden, Department of Biology, Faculty of Science, University of Zagreb, Marulićev trg 9a, \\ HR-10000 Zagreb, Croatia (e-mail: sanja.kovacic@biol.pmf.hr)
}

Kovačić, S.: Plethora of plants - collections of the Botanical Garden, Faculty of Science, University of Zagreb (3): Iris (Iridaceae) Collection. Nat. Croat., Vol. 28, No. 2., 483-514, 2019, Zagreb.

In this paper, the plant lists of the genus Iris (Iridaceae family) grown in Zagreb Botanical Garden of the Faculty of Science since 1895 are studied. Synonymy, nomenclature and origin of plant material were sorted. Lists of species grown in the last 124 years have been constructed to show that during that period at least 273 taxa of wild and cultivated irises inhabited the Garden's collections. Today we have 168 species, cultivars and hybrids.

Key words: Zagreb Botanical Garden, Faculty of Science, historic plant collections, Iris collection

Kovačić, S.: Obilje bilja - zbirke Botaničkoga vrta Prirodoslovno-matematičkog fakulteta Sveučilišta u Zagrebu (3): Zbirka perunika (Iris, Iridaceae). Nat. Croat., Vol. 28, No. 2., 483-514, 2019, Zagreb.

U ovom članku sastavljeni su popisi svojta perunika (rod Iris, porodica Iridaceae) uzgajanih u Botaničkom vrtu zagrebačkog Prirodoslovno-matematičkog fakulteta između 1895. i 2019. godine. Uređena je sinonimika i nomenklatura te istraženo podrijetlo biljnog materijala. Rezultati pokazuju da su tijekom 124 godine kroz zbirke Botaničkog vrta prošle najmanje 273 divlje i uzgojne svojte perunika. Danas uzgajamo 168 vrsta, kultivara i križanaca.

Ključne riječi: Botanički vrt PMF-a u Zagrebu, povijesne zbirke biljaka, rod Iris

\section{INTRODUCTION}

The comprehensive investigation of plant collections in the Botanical Garden of the Faculty of Science, University of Zagreb (in further text "Botanical Garden" or "the Garden") initiated in 2012 is continuing with inventories of indigenous, wild and cultivated taxa of the genus Iris Tourn. ex L. (Iridaceae family). The general facts about the Garden and its collections were published in the first paper of this series (Kovačıć, 2015) and will not be repeated here. Following the established principles, I made an inventory of the genus Iris s.l. taxa cultivated in collections of our Botanical Garden since 1895.

Irises are well known and extensively cultivated around the globe for centuries and are used in perfumery and pharmacy. The genus Iris includes between 200 and 300 species, depending on the comprehension of the genus range (BARKER \& Govaerts, 2016); for example, more than 1400 taxa - species, subspecies, natural varieties and hybrids-are listed in The Plant List database (http://www.theplantlist. 
org/), and its successor World Flora Online (http://www.worldfloraonline.org/), and about 1500 in the databases of cultivated taxa (for example, the Pacific Bulb Society, https://www.pacificbulbsociety.org/pbswiki/index.php/Iris). Wild taxa are divided into subgenera, sections and subsections (SHEAR, 2002), inter-relations of which are complex and not included in this inventory. Most Iris species are distributed in the temperate regions of Europe, Asia and North America, predominantly in dry, semi-desert, or rocky mountainous areas, less in grasslands, meadowlands, bogs and riverbanks (Камалатsку \& Окиво, 2012). Iris species, cultivars and hybrids grown in the collections of our Botanical Garden during the years have been planted outdoors, where they are represented by several specimens each, and are periodically rejuvenated by being divided (vegetatively). Irises are not listed in the CITES-lists (http://checklist.cites.org/\#/en), but up to 26 taxa are listed as (potentially) invasive acc. to EASIN (http://alien.jrc.ec.europa. eu/SpeciesMapper), though of low/unknown impact.

Samples for the wild taxa collections in our Garden are obtained through the Delectus (Index) Seminum-network of inter-botanic-garden seed exchange, while the collections of cultivars and hybrids are supplemented from specialist nurseries or private collectors. Some of the estimations calculate that up to 100000 Iris cultivars are available today in innumerable nurseries around the world, while around 41400 are registered by name with the general authority, the American Iris Society (https://irises.org/), presenting this genus as the most popular monocotyledon in cultivation today. These are reasons for the endangerment of some Iris taxa in their natural habitats. More than 20 wild Iris species - especially those very rare, aesthetically pleasing or/and parental to the vast selection of horticultural hybrids and cultivars - are today red-listed. The Global Red List (https://www.iucnredlist.org) holds seven Iris species: one critically endangered $(\mathrm{CR})$, four endangered (EN) and two vulnerable (VU). European, and combined Euro-Mediterranean Red Lists hold a single critically endangered (CR) Iris species each, while the Mediterranean Red List contains 14 species: 4 (CR), 6 (EN) and 4 (VU). Many more Iris taxa are listed as data deficient (DD) or estimated as of low concern (LC) in all of the aforementioned Red Lists (https://www.iucnredlist.org). The Croatian Red List (https://hirc.botanic.hr/fcd/CrvenaKnjiga/) and subsequent legislation hold two vulnerable (VU) Iris taxa, both - interestingly - unrecognized by the "higher authorities" (for example, Euro+Med Plantbase, World Flora Online, Global Red List, etc.). These are the Croatian "local endemics" (Mrtić \& Cigić, 2007) Iris croatica Horvat \& M. D. Horvat (Miтić et al., 2004) and I. sibirica L. subsp. sibirica (TopIć et al., 2004). The entire Iris genus, with 14 indigenous species, two subspecies and two natural hybrids, is statutorily strictly protected in all Croatian wild habitats (http://www.propisi.hr/print.php?id=12728). It is worth mentioning that irises are closely related to the Slavic mythology: the Croatian (and not only Croatian) word for the genus, "perunika", dedicates its flower to the highest deity of the ancient Slavic pantheon - the thunder god Perun. 


\section{MATERIAL \& METHOD}

Three main sources are used for the construction of lists of irises that have grown in the Botanical Garden's collections since its foundation. As explained in Kovačić (2015), these are: (i) published records on the historic collections of the early days (1891-1896), (ii) a passive database of plants cultivated in the Garden since the early 1950s, but not living today and (iii) an active database of plants recently (September 2019) living in our collections.

The initial part of this study (i) is based on a booklet written by the founder of our Botanical Garden, Professor Antun Heinz (HeInz, 1895-1896), often imprecise in stating the details on the inventory. After that, there is a gap of more than 50 years during which the data on the Garden collections are missing - until recent records were founded in the early 1950s, and since than systematically gathered (details in Kovačıć, 2015). Unlike the glasshouse exotics described in our previous papers (Kovačić, 2015; SANDEv et al., 2017), the origin of most of the Iris species in our inventory cards is well documented. Part of the reason lies, possibly, in the fact that irises appear relatively late in our inventory cards (late 1950s), when the protocol was already well established and new taxa ordered via the Index Seminum network, or brought in from field research. For most of the entries the plant origin is clearly noted (hometown, botanic garden, natural site, donator, or collector), together with a year when the material was acquired, and in which form (seed, cutting, bulb, rhizome or living plant - planta viva).

As the irises are grown in several Garden collections, I divided the inventory lists of taxa in four groups. These informal, and partly overlapping groups are:

1. indigenous Iris-species of wild origin, brought to the Garden from field excursions as living plants (plantae vivae) and further grown in the collections (phytogeographical sections or rockeries) dedicated to the native flora (Tab. 1, Photo-table 1);

2. other, non-native Iris species of presumably wild origin, obtained via Index Seminum publications, grown from the seeds and planted elsewhere in the Garden (Tab. 2, Photo-table 2);

3. cultivars of wild species partly acquired as living plants (incl. rhizomes and bulbs), partly grown from seeds obtained via Index Seminum publications, out of which some could be presumably of wild origin (natural varieties and forms, rarely hybrids), but are mostly of garden-origin; planted in the Garden's ornamental flowerbeds, nurseries or systematic fields (Tab. 3, Photo-table 3);

4. Iris Barbata hybrids (also called "bearded" irises, mostly of the "tall bearded" group, an offspring of the Iris $\times$ germanica and I. variegata progenitors), plants of elaborated man-made origin achieved via meticulous crossings of vast numbers of named and un-named (called "seedlings") hybrids. These are acquired strictly as living plants (in the form of rhizomes) and planted in the Garden's nurseries, ornamental flowerbeds and Iridarium, established in 2004 (Tab. 4, Photo-table 4).

As our Botanical Garden grows many "local" plants of South East European distribution, I felt that the authentic nomenclature as stated in our old inventorycards - even though today partly unrecognized - should be preserved. Therefore, 
Tables 1 and 2 are designed to conserve the original plant names, the entries by which the specimens arrived to our collection, whenever possible: unfortunately, sometimes the original names during the years were erased and replaced with the valid synonyms of that time. Recently valid, up-do-date nomenclature is added in the last columns of Tabs. 1 and 2, when possible: in some occasions it was not possible to connect the original name-entry to the recently valid name.

Therefore, the nomenclature in this paper follows several authorities, in accordance with the group of irises in focus, in attempt to keep the historical data with up-to-date synonyms.

In Tab. 1, the main authority is the Croatian Flora Database (https://hirc.botanic. $\mathrm{hr} / \mathrm{fcd} /$ ), for the sole reason that many Croatian Iris-taxa are not recently recognized by the prominent floristic databases (Euro+Med Plantbase, World Flora Online, IPNI). If some taxon is not included in the Croatian flora, the Euro+Med Plantbase is consulted secondarily (http://ww2.bgbm.org/EuroPlusMed/query.asp). The World Flora Online (http://www.worldfloraonline.org/) database is added last, also used as the nomenclatural authority in the Tabs. 2 and 3 for the species outside Euro+Med Plantbase's range. Names of cultivars and hybrids in the Tab. 3 follow botanical nomenclature whenever possible; but also other, "less scientific" sources, such as horticultural databases. For the Iris Barbata ("bearded") group of hybrids listed in Table 4, the Iris Encyclopedia of the American Iris Society (http:// wiki.irises.org/) was consulted first, followed by the databases of the Historic Iris Preservation Society (https://www.historiciris.org/) and the National Gardening Organization (https:/garden.org/plants/view/181474/Irises-Iris/).

\section{RESULTS \& DISCUSSION}

As seen in Heinz (1895-1896), in the late 19th century only four Iris species are named as living in our Botanical Garden, all "common Croatian" taxa that we still grow today. The Iris genus is described very poorly, as "in sizable number" (..."oveći broj Iris-vrsta, perunika...") of "mostly Mediterranean species", highlighting by name only "medicinal species" Iris germanica, I. pallida and I. florentina, besides I. pseudacorus (HeInz, 1895-1896 - p. 23). Two of these kept their original names: the common yellow flag (Iris pseudacorus L.) and the Dalmatian iris (I. pallida Lam., which include I. illyrica Tomm. and I. pseudopallida Trinajstić, still recognized by the Flora Croatica Database), most probably originally endemic to the Croatian coast, but widely cultivated elsewhere. The blue German bearded iris (I. germanica L.) and white German bearded iris or Florentine iris ( $I$. florentina L.) are today nested in the range of an olden cultivated "megataxon", Iris $\times$ germanica L., which includes many "little" taxa considered to be endemic in this part of Europe (Mitić \& Cigić, 2007). Today we do not know where those first irises were planted in the Garden (systematic fields, beds with the "useful plants", flowerbeds?), except for I. pseudacorus ("growing by the pond"). Also, we do not know where those plants originated: were they from Croatian wild localities, or of some "other-garden" origin? 


\section{Indigenous or wild irises collected in Croatia and the neighbourhood countries}

Tab. 1 comprises the irises collected in their natural habitats, which were (and still are) grown in the national indigenous plant collections ("the rockeries") in our Garden: phytogeographical sections (Karstic - K, Mediterranean - M and sub-Mediterranean - SBM), established during the "Second Yugoslavia" era (1945 - 1991). Details about the Garden's rockeries with indigenous flora could be found in Stamenković \& Kovačić (2014).

According to my calculations, since 1959 at least 24 Iris taxa have passed through the Garden phytogeographical sections, out of which we keep 14 today (Tab. 1, Photo-table 1). It must be emphasized that several taxa were never properly determined before vanishing from the collections (inventory-cards designated as "Iris sp." were excluded from this inventory). Also, the samples assigned "for scientific purposes" (in Tabs. 1 and 2) are not parts of the Garden's collection, but kept in our nurseries for the research of Professor Božena Mitić and her colleagues.

Most of the samples (51) listed in Tab. 1 were collected in Croatian localities as living plants, while 12 originate from neighbouring countries (Bosnia and Herzegovina, Serbia and North Macedonia that were during the time of collecting, like Croatia, part of Yugoslavia). Six samples are of unknown origin ("nn" in Tab. 1 and other tables). Older scientific names are difficult to track, due to synonymy and taxonomical changes, so I cannot be absolutely certain about which taxon some of the plant samples, now missing from our collections, actually belonged to. As already emphasized, I intentionally kept the original plant-names from our inventory-cards of the plants today missing from our collection, to preserve this vivid, today often neglected, nomenclature of the past. It is also worth mentioning that some of the samples planted in the rockeries were collected as wild-growing, but are today considered to be of an ancient cultivated origin: that is particularly the case with the "local taxa" such as blue and white German bearded irises (I. × germanica and I. × florentina), which were grown as "indigenous" in our phytogeographical sections (rockeries).

As Tab. 1 shows, the first irises from the wild were brought to the Garden in 1959, by our first post-WWII Garden Manager Dr. Sala Ungar. Those were Iris illyrica Tomm. (I. illyrica Tomm. ex Vis. is today considered to be a synonym of I. pallida Lam. subsp. illyrica (Tomm. ex Vis.) K. Richt.) from the Croatian North Adriatic shores (Sušanj near Karlobag); I. macedonica Horv. (illegitimate name, probably one of the $I$. reichenbachii Heuff. range) and I. pumila L. subsp. attica (Boiss. et Heldr.) Hay. f. ochridana (unknown form) from North-Macedonian Mt Galičica. The others were brought mostly as living plants (planta viva) from Dr. Ungar's field excursions across ex-Yugoslavia in the 1960s.

According to the national Flora Croatica Database, the Croatian flora today comprises 14 Iris species (https://hirc.botanic.hr/fcd/ShowResults. aspx?hash=-1000434997), out of which several are included in the larger ("mega"-) taxa according to prominent botanic databases (Euro+Med 
Tab. 1. Indigenous Iris species native to Croatia and neighbouring countries, brought from the field excursions as living plants and grown in the collections of native plants in the Botanical Garden from 1959 to 2019.

\begin{tabular}{|c|c|c|c|c|c|c|c|c|c|}
\hline $\begin{array}{l}\text { Scientific name } \\
\text { (from the original } \\
\text { inventory-card) }\end{array}$ & $\begin{array}{l}\text { Origin of plant } \\
\text { material }^{*}\end{array}$ & $\begin{array}{l}\text { Year of } \\
\text { obtaining }\end{array}$ & $\begin{array}{l}\text { Last } \\
\text { recorded }\end{array}$ & Collection & $\begin{array}{l}\text { Notes in } \\
\text { the original } \\
\text { inventory-card }\end{array}$ & FCD & Euro+Med & Plant List & $\begin{array}{l}\text { Notes and } \\
\text { remarks }\end{array}$ \\
\hline $\begin{array}{l}\text { Iris adriatica } \\
\text { Trinajstić }\end{array}$ & Čiovo & 1988 & 1989 & M & \multirow{4}{*}{$\begin{array}{l}\text { I. adriatica } \\
\text { Trinajstić ex } \\
\text { Mitić }\end{array}$} & \multirow{5}{*}{\multicolumn{3}{|c|}{ Iris adriatica Trinajstić ex Mitić }} & \multirow{5}{*}{$\begin{array}{l}\text { author of the } \\
\text { valid taxon } \\
\text { description, } \\
\text { Professor Mitić, } \\
\text { is keeping her } \\
\text { scientific samples } \\
\text { in the Garden }\end{array}$} \\
\hline & Šibenik & 1988 & 1989 & M & & & & & \\
\hline & Bibinje & 1988 & 1989 & M & & & & & \\
\hline & Unešić & 2001 & 2001 & Nursery & & & & & \\
\hline & Bilice & 2016 & 2019 & Nursery & $\begin{array}{l}\text { for scientific } \\
\text { purposes }\end{array}$ & & & & \\
\hline Iris bosniaca Beck & $\begin{array}{l}\text { Zelengora, } \\
\text { Bosnia \& } \\
\text { Herzegovina }\end{array}$ & 1966 & 1989 & SBM & $\begin{array}{l}\text { I. bosniaca } \\
\text { (Beck) Dörfl. }\end{array}$ & no & no & $\begin{array}{l}\text { I. reichenbachii } \\
\text { Heuff. }\end{array}$ & \\
\hline Iris cengialti Ambr. & Krk & 1967 & 1979 & M & syn. I. pallida & \multirow{3}{*}{$\begin{array}{l}\text { I. cengialti } \\
\text { Ambr. is a } \\
\text { synonim of } \\
\text { I. illyrica Tomm. }\end{array}$} & \multirow{3}{*}{\multicolumn{2}{|c|}{$\begin{array}{l}\text { I. cengialti Ambrosi ex } \\
\text { A.Kern. is a synonim of } \\
\text { I. pallida subsp. cengialti } \\
\text { (Ambrosi ex A.Kern.) } \\
\text { Foster }\end{array}$}} & corr. I. illyrica \\
\hline & Gornje Jelenje & 1965 & 2012 & SBM & $\begin{array}{l}\text { cengialti } \\
\text { (Ambr.) Foster }\end{array}$ & & & & corr. I. pallida \\
\hline & nn & 1993 & 2019 & Nursery & $\begin{array}{l}\text { for scientific } \\
\text { purposes }\end{array}$ & & & & \\
\hline \multirow[t]{3}{*}{ Iris croatica Horv. } & $\begin{array}{l}\text { Samoborsko } \\
\text { gorje }\end{array}$ & 1963 & 1989 & $\mathrm{~K}$ & \multirow{3}{*}{$\begin{array}{l}\text { I. croatica } \\
\text { Horvat et } \\
\text { Horvat M. }\end{array}$} & \multirow{3}{*}{$\begin{array}{l}\text { I. croatica } \\
\text { Horvat et } \\
\text { M. D. Horvat } \\
\text { VU }\end{array}$} & \multirow{3}{*}{ no } & \multirow{3}{*}{$\begin{array}{l}\text { I. } \times \text { croatica } \\
\text { Horvat \& } \\
\text { M.D.Horvat / } \\
\text { illegitimate/ is } \\
\text { a synonym of } \\
\text { I. } \times \\
\text { germanica L. }\end{array}$} & \\
\hline & Strahinjčica & 1970 & 2019 & K & & & & & \\
\hline & Medvednica & 1989 & 2019 & K & & & & & \\
\hline \multirow[t]{6}{*}{ Iris florentina L. } & nn & 1962 & 2019 & $\begin{array}{l}\text { Systematic } \\
\text { fields }\end{array}$ & & \multirow{6}{*}{$\begin{array}{l}\text { Iris germanica } \\
\text { L. }\end{array}$} & \multirow{6}{*}{ no } & \multirow{6}{*}{$\begin{array}{l}\text { unresolved } \\
\text { name }\end{array}$} & \multirow{6}{*}{$\begin{array}{l}\text { I. germanica } \\
\text { nothovar. } \\
\text { florentina or } \\
\text { "Iris Florentina" } \\
\text { (www.rhs.org.uk) } \\
\text { I. x florentina } \\
\text { is incl. to I. × } \\
\text { germanica group }\end{array}$} \\
\hline & nn & 1963 & 1969 & Nursery & & & & & \\
\hline & Orebić & 1969 & 2001 & M & & & & & \\
\hline & nn & 2008 & 2019 & Nursery & $\begin{array}{l}\text { (for scientific } \\
\text { purposes) }\end{array}$ & & & & \\
\hline & Orebić & 2017 & 2019 & Iridarium & & & & & \\
\hline & Orebić & 1969 & 2001 & $\begin{array}{l}\text { Nursery, } \\
\text { later M }\end{array}$ & & & & & \\
\hline \multirow[t]{4}{*}{ Iris graminea L. } & $\begin{array}{l}\text { Samoborsko } \\
\text { gorje }\end{array}$ & 1961 & 2010 & $\mathrm{~K}$ & & \multirow{4}{*}{\multicolumn{3}{|c|}{ accepted }} & \\
\hline & Velebit & 1963 & 2019 & $\mathrm{~K}$ & & & & & \\
\hline & Klek & 1999 & 2019 & K & & & & & \\
\hline & Ravna gora & 1978 & 1989 & Nursery & & & & & \\
\hline \multirow[t]{9}{*}{ Iris illyrica Tomm. } & Plomin & 1965 & 2019 & M & \multirow{9}{*}{$\begin{array}{l}\text { "syn. I. } \\
\text { cengialti Ambr. } \\
\text { subsp. illyrica } \\
\text { (Tomm.) Pemp. } \\
\text { - invalid!" }\end{array}$} & \multirow{9}{*}{ accepted } & \multirow{9}{*}{ no } & \multirow{9}{*}{$\begin{array}{l}\text { I. illyrica } \\
\text { Tomm. ex Vis. } \\
\text { is a synonym of } \\
\text { I. pallida subsp. } \\
\text { illyrica (Tomm. } \\
\text { ex Vis.) K.Richt. }\end{array}$} & \\
\hline & Krk & 1967 & 1981 & M & & & & & \\
\hline & Vir & 1966 & 1989 & M & & & & & \\
\hline & Biokovo & 1967 & 2019 & SBM & & & & & \\
\hline & Sušanj & 1959 & 1963 & $\mathrm{~K}$ & & & & & \\
\hline & Velebit & 1974 & 1986 & K & & & & & \\
\hline & Velebit & 1975 & 2001 & K & & & & & \\
\hline & Velebit & 1983 & 1986 & $\mathrm{~K}$ & & & & & \\
\hline & Velebit & 1994 & 2019 & $\mathrm{~K}$ & & & & & \\
\hline $\begin{array}{l}\text { Iris macedonica } \\
\text { Horv. (?) }\end{array}$ & $\begin{array}{l}\text { Galičica, North } \\
\text { Macedonia }\end{array}$ & 1959 & 1984 & SBM & $\begin{array}{l}\text { 'I.m. Nadji', or } \\
\text { 'I.m. Heldr. ?' }\end{array}$ & no & & & $\begin{array}{l}\text { Probably from } \\
\text { the range of I. } \\
\text { reichenbachii }\end{array}$ \\
\hline
\end{tabular}




\begin{tabular}{|c|c|c|c|c|c|c|c|c|c|}
\hline $\begin{array}{l}\text { Scientific name } \\
\text { (from the original } \\
\text { inventory-card) }\end{array}$ & $\begin{array}{l}\text { Origin of plant } \\
\text { material* }\end{array}$ & $\begin{array}{l}\text { Year of } \\
\text { obtaining }\end{array}$ & $\begin{array}{l}\text { Last } \\
\text { recorded }\end{array}$ & Collection & $\begin{array}{l}\text { Notes in } \\
\text { the original } \\
\text { inventory-card }\end{array}$ & FCD & Euro+Med & Plant List & $\begin{array}{l}\text { Notes and } \\
\text { remarks }\end{array}$ \\
\hline \multirow[t]{6}{*}{ Iris pallida Lam. } & $\begin{array}{l}\text { Radika, North } \\
\text { Macedonia }\end{array}$ & 1963 & 2019 & SBM & & \multirow{6}{*}{\multicolumn{3}{|c|}{ accepted }} & \\
\hline & Dubrovnik & 1983 & 1989 & M & $\begin{array}{l}\text { prob. I. } \\
\text { pseudopallida }\end{array}$ & & & & \\
\hline & Biokovo & 1962 & 1989 & SBM & & & & & \\
\hline & Dinara & 1982 & 1963 & SBM & & & & & \\
\hline & Biokovo & 1964 & 1988 & SBM & & & & & \\
\hline & Mt St. Ilija & 1971 & 2019 & SBM & $\begin{array}{l}\text { prob. I } \\
\text { pseudopallida }\end{array}$ & & & & \\
\hline \multirow[t]{4}{*}{$\begin{array}{l}\text { Iris pallida } \\
\text { Lam. subsp. } \\
\text { dalmatica Pamp. } \\
\text { (I. pseudopallida } \\
\text { Trinajstić) }\end{array}$} & Bobara Isle & 1972 & 1974 & Nursery & $\begin{array}{l}\text { I. pallida Lam. } \\
\text { subsp. illyrica } \\
\text { (Tomm. ex } \\
\text { Vis.) K. Richt. }\end{array}$ & \multirow{4}{*}{$\begin{array}{l}\text { I. pallida Lam. } \\
\text { var. dalmatica } \\
\text { Pamp. is a } \\
\text { synonim of I. } \\
\text { pseudopallida } \\
\text { Trinajstić }\end{array}$} & \multirow{4}{*}{\multicolumn{2}{|c|}{$\begin{array}{l}\text { I. pallida var. dalmatica } \\
\text { Pamp. is a synonym of } \\
\text { I. pallida subsp. illyrica } \\
\text { (Tomm. ex Vis.) K.Richt. }\end{array}$}} & \\
\hline & Korčula Island & 1987 & 2019 & M & Pamp. = I. & & & & \\
\hline & \begin{tabular}{|l} 
Pelješac \\
Peninsula
\end{tabular} & 1992 & 2019 & EuMed & Trinajstić & & & & \\
\hline & $\begin{array}{l}\text { Cetina River } \\
\text { Valley }\end{array}$ & 1999 & 2019 & Nursery & $\begin{array}{l}\text { for scientific } \\
\text { purposes }\end{array}$ & & & & \\
\hline \multirow[t]{4}{*}{ Iris pseudacorus L. } & $\mathrm{nn}$ & 1962 & 2019 & Ponds & & \multirow{4}{*}{\multicolumn{3}{|c|}{ accepted }} & \\
\hline & Jablanovec & 1989 & 2019 & Ponds & & & & & \\
\hline & nn & 2001 & 2019 & Ponds & & & & & \\
\hline & Pisarovina & 2009 & 2019 & Nursery & & & & & \\
\hline \multirow[t]{4}{*}{ Iris pumila L. } & $\mathrm{nn}$ & 1963 & 1963 & Nursery & & \multirow{4}{*}{\multicolumn{3}{|c|}{ accepted }} & \\
\hline & $\mathrm{nn}$ & 1963 & 1978 & K & & & & & \\
\hline & $\begin{array}{l}\text { Deliblato Sands, } \\
\text { Serbia }\end{array}$ & 1965 & 2019 & SBM & & & & & \\
\hline & Bansko brdo & 2013 & 2019 & Nursery & & & & & \\
\hline \multirow[t]{2}{*}{$\begin{array}{l}\text { Iris pumila L. } \\
\text { subsp. attica (Boiss. } \\
\text { et Heldr.) Hay. }\end{array}$} & $\begin{array}{l}\text { Orlovo brdo, N. } \\
\text { Macedonia }\end{array}$ & 1973 & 1980 & Nursery & \multirow[t]{2}{*}{$\begin{array}{l}\text { I. attica Boiss. } \\
\text { et Heldr. }\end{array}$} & \multirow[t]{2}{*}{ no } & \multirow{2}{*}{\multicolumn{2}{|c|}{$\begin{array}{l}\text { I. pumila subsp. attica } \\
\text { (Boiss. \& Heldr.) K.Richt. }\end{array}$}} & \multirow[t]{2}{*}{$\begin{array}{l}\text { (from native } \\
\text { locality) }\end{array}$} \\
\hline & Sarajevo & 1975 & 1980 & Nursery & & & & & \\
\hline $\begin{array}{l}\text { Iris pumila L. } \\
\text { subsp. attica (Boiss. } \\
\text { et Heldr.) "Hay. f. } \\
\text { ochridana" }\end{array}$ & $\begin{array}{l}\text { Galičica, North } \\
\text { Macedonia }\end{array}$ & 1959 & 1973 & SBM & & \multicolumn{3}{|l|}{ no } & unknown forma \\
\hline $\begin{array}{l}\text { Iris pumila L. } \\
\text { "subsp. eupumila f. } \\
\text { violacea Ker." }\end{array}$ & $\mathrm{nn}$ & 1962 & 1963 & K & & \multicolumn{3}{|l|}{ no } & $\begin{array}{l}\text { unknown } \\
\text { subspecies and } \\
\text { forma }\end{array}$ \\
\hline $\begin{array}{l}\text { Iris reginae } \\
\text { Horv. (?) }\end{array}$ & $\begin{array}{l}\text { Galičica, North } \\
\text { Macedonia }\end{array}$ & 1967 & 1984 & Nursery & $\begin{array}{l}\text { incorrect - I. } \\
\text { variegata }\end{array}$ & no & no & \begin{tabular}{|l} 
I. reginae \\
Horvat \& \\
M.D.Horvat is \\
a synonim of I. \\
variegata L. \\
\end{tabular} & \\
\hline \multirow[t]{2}{*}{$\begin{array}{l}\text { Iris } \\
\text { reichenbachii } \\
\text { Heuff. }\end{array}$} & Rtanj, Serbia & 1974 & 1979 & nursery & & \multirow{2}{*}{\multicolumn{3}{|c|}{ accepted }} & \multirow{2}{*}{$\begin{array}{l}\text { includes } \\
\text { many Balkan } \\
\text { "local taxa", } \\
\text { f.e. Iris athoa, } \\
\text { I. balkana, I. } \\
\text { bosniaca, I. } \\
\text { macedonica, I. } \\
\text { serbica... }\end{array}$} \\
\hline & $\begin{array}{l}\text { Kopaonik, } \\
\text { Serbia }\end{array}$ & 1965 & 2019 & SBM & & & & & \\
\hline \begin{tabular}{|l} 
Iris \\
rubromarginata \\
Baker 'f. \\
flavescens Azn.' \\
\end{tabular} & $\begin{array}{l}\text { Galičica, } \\
\text { North } \\
\text { Macedonia }\end{array}$ & 1960 & 1973 & SBM & & no & \multicolumn{2}{|c|}{$\begin{array}{l}\text { val. I. suaveolens Boiss. } \\
\text { \& Reut.; unknown forma }\end{array}$} & \\
\hline $\begin{array}{l}\text { Iris rudsky } \\
\text { Horv. (?) }\end{array}$ & $\begin{array}{l}\text { Sokolac, } \\
\text { Bosnia \& } \\
\text { Herzegovina }\end{array}$ & 1967 & 1984 & Nursery & $\begin{array}{l}\text { incorrect - } \\
\text { I. variegata }\end{array}$ & no & & $\begin{array}{l}\text { I. rudskyi } \\
\text { Horvat \& } \\
\text { M.D.Horvat } \\
=\text { I. } \\
\text { variegata L. }\end{array}$ & \\
\hline
\end{tabular}




\begin{tabular}{|c|c|c|c|c|c|c|c|c|c|}
\hline $\begin{array}{l}\text { Scientific name } \\
\text { (from the original } \\
\text { inventory-card) }\end{array}$ & $\begin{array}{l}\text { Origin of plant } \\
\text { material* }^{*}\end{array}$ & $\begin{array}{l}\text { Year of } \\
\text { obtaining }\end{array}$ & $\begin{array}{l}\text { Last } \\
\text { recorded }\end{array}$ & Collection & \begin{tabular}{|l|} 
Notes in \\
the original \\
inventory-card
\end{tabular} & FCD & Euro+Med & Plant List & $\begin{array}{l}\text { Notes and } \\
\text { remarks }\end{array}$ \\
\hline \multirow[t]{4}{*}{ Iris sibirica L. } & nn & 1962 & 2019 & $\begin{array}{l}\text { Systematic } \\
\text { fields }\end{array}$ & & \multirow{6}{*}{ accepted } & \multirow{4}{*}{\multicolumn{2}{|c|}{ accepted }} & \multirow{6}{*}{$\begin{array}{l}\text { acc. to FCD Iris } \\
\text { sibirica includes } \\
\text { two subspecies: I } \\
\text { sibirica L. subsp. } \\
\text { sibirica (VU) } \\
\text { and I. sibirica L. } \\
\text { subsp. erirrhiza } \\
\text { (Posp.) Wraber. }\end{array}$} \\
\hline & Platak & 1968 & 1973 & SBM & $\begin{array}{l}\text { arrived as I. } \\
\text { graminea }\end{array}$ & & & & \\
\hline & $\mathrm{nn}$ & 1987 & 1988 & Nursery & $\begin{array}{l}\text { arrived as I. } \\
\text { delavayi }\end{array}$ & & & & \\
\hline & Risnjak & 1965 & 1993 & SBM & & & & & \\
\hline $\begin{array}{l}\text { I. sibirica L. subsp. } \\
\text { sibirica }\end{array}$ & Petrijevci & 2004 & 2019 & $\begin{array}{l}\text { Systematic } \\
\text { fields }\end{array}$ & $\begin{array}{l}\text { for scientific } \\
\text { purposes }\end{array}$ & & \multirow[b]{2}{*}{ no } & \multirow[b]{2}{*}{ no } & \\
\hline $\begin{array}{l}\text { I. sibirica L. subsp. } \\
\text { erirrhiza (Posp.) } \\
\text { Wraber }\end{array}$ & Bjelolasica & 2012 & 2019 & Nursery & $\begin{array}{l}\text { for scientific } \\
\text { purposes }\end{array}$ & & & & \\
\hline $\begin{array}{l}\text { Iris } \\
\text { (Hermodactylus) } \\
\text { tuberosa L. }\end{array}$ & Zaton-Doli & 2001 & 2019 & SBM & \begin{tabular}{|l} 
Hermodactylus \\
tuberosus (L.) \\
Mill.
\end{tabular} & $\begin{array}{l}\text { Hermodactylus } \\
\text { tuberosus (L.) } \\
\text { Mill. }\end{array}$ & \multicolumn{2}{|l|}{ I. tuberosa L. } & \\
\hline \multirow[t]{8}{*}{ Iris variegata $\mathrm{L}$. } & nn & 1962 & 1969 & K & & \multirow{8}{*}{ accepted } & & & \\
\hline & $\begin{array}{l}\text { Deliblato Sands, } \\
\text { Serbia }\end{array}$ & 1965 & 1989 & SBM & & & & & \\
\hline & Dinara & 1966 & 1969 & Nursery & & & & & \\
\hline & Velebit & 1968 & 1969 & K & & & & & \\
\hline & Papuk & 1969 & 1989 & K & & & & & \\
\hline & Klek & 1970 & 1970 & Nursery & & & & & \\
\hline & Lička Plješivica & 1974 & 1975 & K & & & & & \\
\hline & Velebit & 1995 & 1997 & K & & & & & \\
\hline
\end{tabular}

*if not stated differently, the locality of plant material collection is situated in Croatian national territory

Plantbase, World Flora Online), or are considered being garden escapees from ancient times. For example, Iris bosniaca (Beck) Dörfl. is today included in the range of I. reichenbachii Heuff.; I. croatica Horvat et M. D. Horvat, I. pseudopallida Trinajstić and I. germanica L. are unrecognized by Euro+Med Plantbase, as are the natural hybrids I. $\times$ rotschildii Degen (I. illyrica Tomm. $\times$ I. variegata L.) and I. $\times$ sambucina L. (I. pallida Lam. $\times$ I. variegata L.). Also, according to Flora Croatica Database, I. sibirica L. in Croatia exists in the form of two subspecies: I. sibirica L. subsp. erirrhiza (Posp.) Wraber (endemic to Croatia and Slovenia) and I. sibirica L. subsp. sibirica. The latter is considered to be vulnerable (VU) according to the Croatian Red Book, even though it is not recognized by the aforementioned plant authorities - first and foremost, the Euro+Med Plantbase. However, World Flora Online, successor of the "garden-friendly" Plant List database, recognizes some of this historic nomenclature that could be seen in the Tables 1 and 2. Nevertheless, some plant names from our inventory cards are today "lost": for example, Iris variegata L. comprises both I. reginae Horvat \& M. D. Horvat and I. rudskyi Horvat \& M. D. Horvat, described by the famous botany professor of our faculty, Dr Ivo Horvat (1897 - 1963) and his wife Marija. Both botanists also described the Croatian "national flower" I. croatica Horvat $\&$ M. D. Horvat, a taxon that has recently become not only unrecognized by "reputable" botanical databases (today it is included in I. × germanica), but also apparently illegitimate, due to the priority of plant naming (see $I . \times$ croatica Prodán in The Plant List and World Flora Online). 


\section{Wild or "natural" irises grown from seed obtained via Index Seminum publications}

Tab. 2 contains the list of irises grown predominantly from seeds obtained via Index Seminum publications. Although the taxa in Tab. 2 are not listed as cultivars, most of them originate from garden growth, so, consequently, some of them could have been hybrids or horticultural varieties - at least, results of the "open pollination" (as frequently stated in the Index Seminum publications), rather than wild species sensu stricto. Tab. 2 depicts 66 taxa of irises that we grew during the last 60 years, out of which we today hold 25 (some can be seen in the Photo-table 2). These were, and still are, grown mostly in several of the Garden nurseries, flowerbeds with horticultural plants or the "Alpinum": the oldest rockery in the Garden, intended for horticultural varieties. For 14 samples the source is unknown (" $\mathrm{nn}$ " in Tab. 2). One Iris rubromarginata Baker subsp. mellita (Janka) K. Richt. (valid I. suaveolens Boiss. \& Reut.), obtained from Vienna in 1953, was the first species of this genus inventoried in the new Garden database, followed by I. crocea Jacquem. ex R.C.Foster (from Nancy, 1955) and I. japonica Thunb. (from Nanking, 1959). It is interesting to see that some species were ordered repeatedly from different botanic gardens during the years, for example, I. kaempferi Siebold ex Lem., 17 times since 1961.

Tab. 2. Wild Iris species, not-native to Croatia, grown from seeds in the Botanical Garden from 1955 to 2019

\begin{tabular}{|c|c|c|c|c|c|c|}
\hline $\begin{array}{l}\text { Scientific name from the original } \\
\text { inventory-card }\end{array}$ & $\begin{array}{l}\text { Origin } \\
\text { (botanical } \\
\text { garden, city, } \\
\text { nursery) }\end{array}$ & $\begin{array}{l}\text { Year of } \\
\text { obtaining }\end{array}$ & \begin{tabular}{|l} 
Last \\
recorded
\end{tabular} & Collection & $\begin{array}{l}\text { Notes in the original } \\
\text { inventory-card }\end{array}$ & $\begin{array}{l}\text { Nomenclature acc. to Euro+Med } \\
\text { for the respective species; acc. to the } \\
\text { Plant List for the rest of the world }\end{array}$ \\
\hline \multirow[t]{5}{*}{ Iris aphylla L. } & Bruxelles & 1960 & 1963 & Nursery & & \multirow{5}{*}{ valid } \\
\hline & Toronto & 1960 & 1963 & Nursery & & \\
\hline & Uppsala & 1971 & 1974 & Nursery & & \\
\hline & Dresden & 1975 & 1977 & Nursery & & \\
\hline & Edmonton & 1985 & 2019 & Alpinum & & \\
\hline Iris biglumis Vahl & Halle & 1996 & 1996 & Nursery & syn. I lactea & valid \\
\hline $\begin{array}{l}\text { Iris bismarckiana Dammen et } \\
\text { Sprenger }\end{array}$ & Athens & 1984 & 1989 & Nursery & syn. I. nazarena Hort. & valid \\
\hline \multirow[t]{2}{*}{ Iris bucharica Foster } & Tashkent & 1973 & 1980 & Nursery & \multirow{2}{*}{$\begin{array}{l}\text { arrived as Juno } \\
\text { bucharica }\end{array}$} & \multirow{2}{*}{ valid } \\
\hline & Athens & 1981 & 2019 & Alpinum & & \\
\hline \multirow[t]{3}{*}{ Iris bulleyana Dykes } & Chelsea & 1960 & 1969 & Nursery & & \multirow{3}{*}{ valid } \\
\hline & Jena & 1983 & 1983 & Nursery & & \\
\hline & Sofia & 1984 & 1985 & Glasshouse & & \\
\hline
\end{tabular}




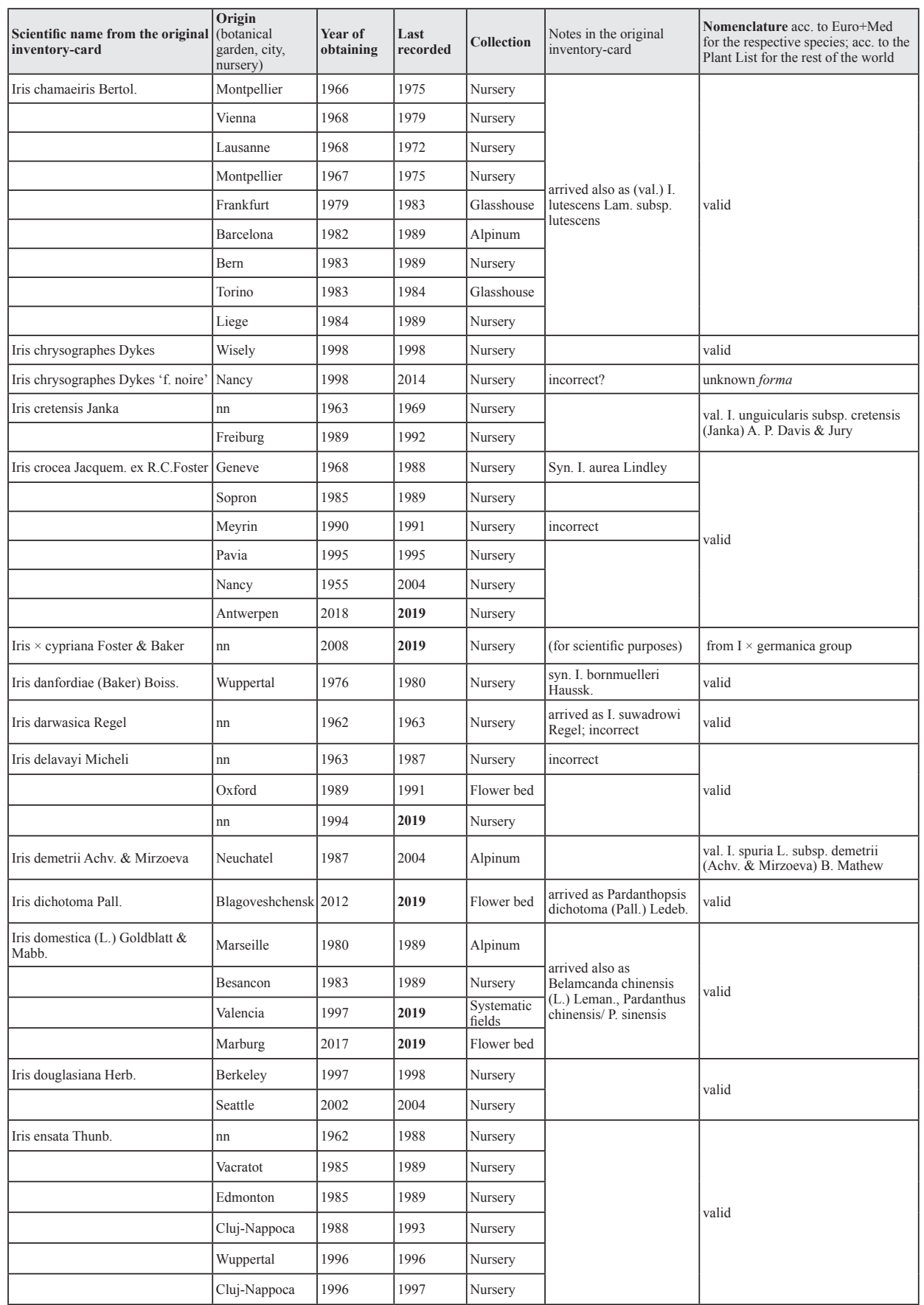




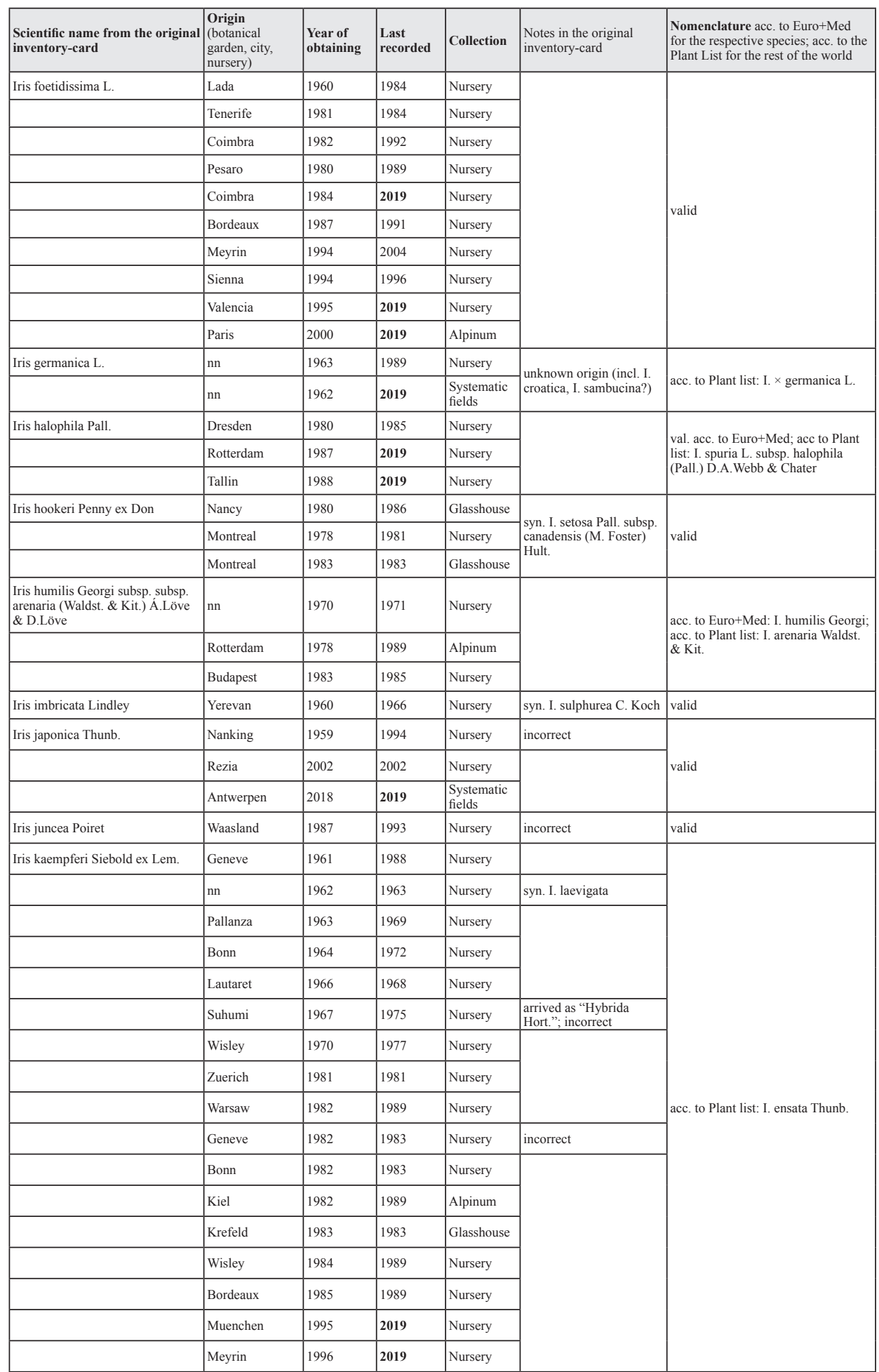




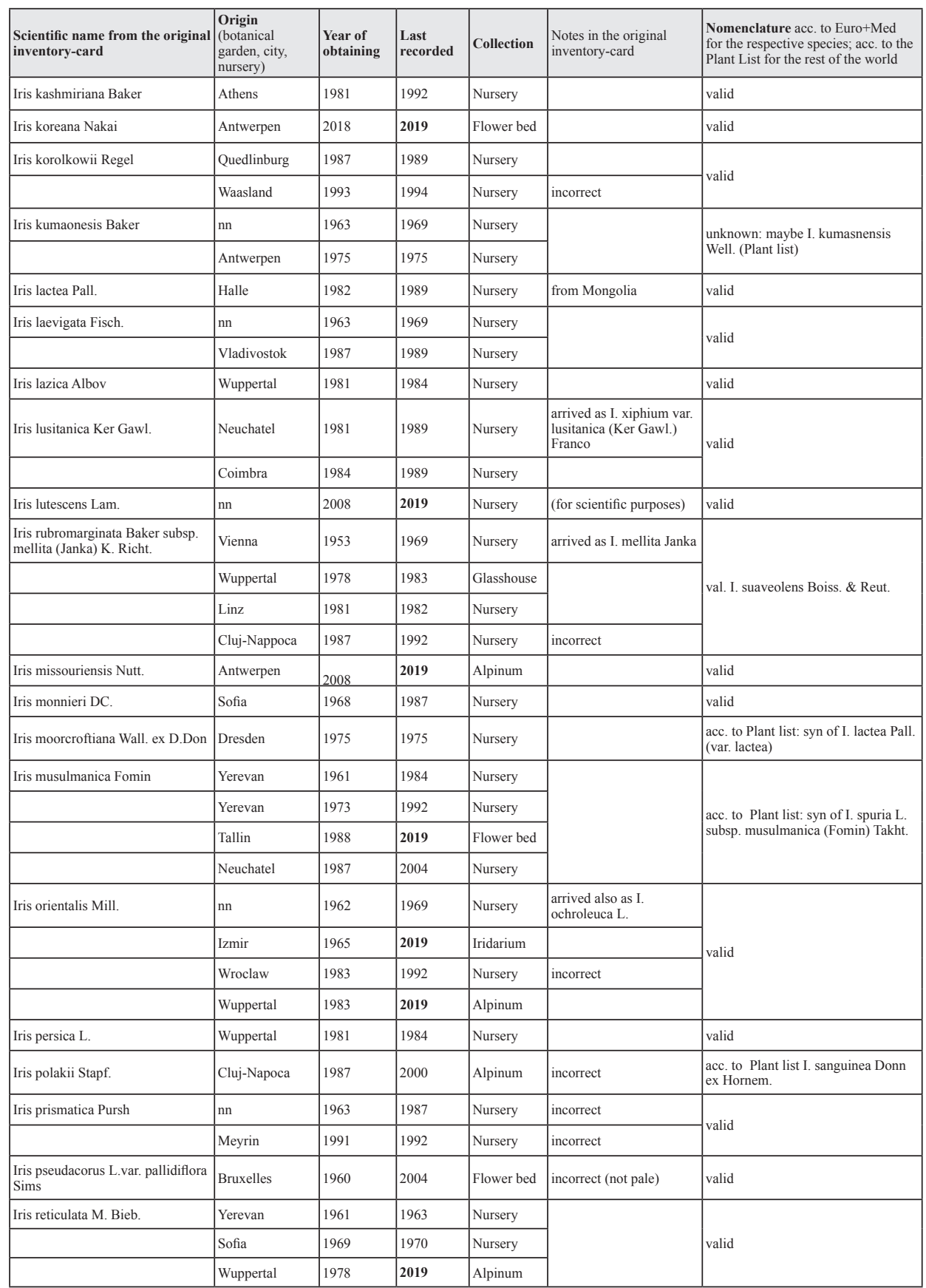




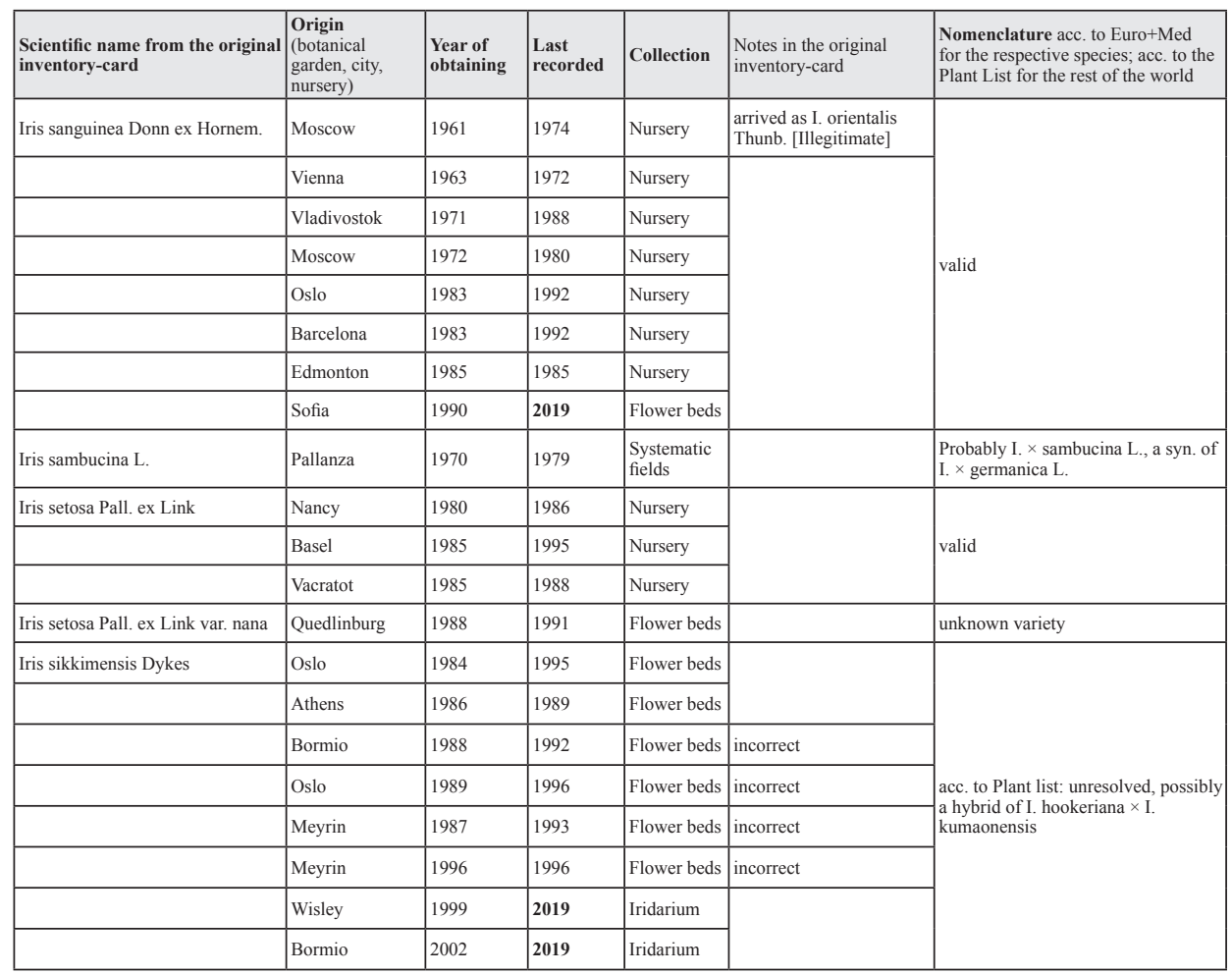

\section{3. "Simple" garden varieties and cultivars of known ancestry}

Various Iris-cultivars were obtained through the years in the form of living plants (planta viva, incl. rhizomes and bulbs), but also grown from seeds obtained via Index Seminum publications. These were, and still are, planted in the flowerbeds of our horticultural displays. As seen in Tab. 3, during the investigated time (since 1963) we had at least 15 named cultivars, out of which today we grow 8 (Photo-table 3). The earliest noted cultivars of this informal group are Iris pallida L. 'Variegata' and the cultivars of I. reticulata M. Bieb. ("Hyb."), gained in 1963 as gifts from person(s) today unknown.

It is worth mentioning that the seed from the plant varieties of garden origin is "expected" to sprout in "unexpected" - but sometimes interesting - forms, due to the open-air pollination and instability of morphologic/phenotypic traits (as for the irises, the colour(s) and size of the flowers, stripes of the leaves and general height of the plant). Sometimes we keep those plants, though "incorrect" (Tab. 3), and yet they are more similar to the wild species than to the named cultivar (for example, our I. sibirica L. 'Phosphorflamme', grown from the seeds, had no "phosphor flame" in it, so we replaced it with a living plant, courtesy of the colleagues from Riga University Botanical Garden). 
Tab. 3. Cultivated varieties of different Iris species, grown in the Botanical Garden from 1960 to 2019.

\begin{tabular}{|c|c|c|c|c|}
\hline Iris - other cultivars: & $\begin{array}{l}\text { Origin and year of } \\
\text { obtaining* }\end{array}$ & $\begin{array}{l}\text { Last } \\
\text { recorded }\end{array}$ & Collection & Notes and remarks \\
\hline \multirow[t]{2}{*}{$\begin{array}{l}\text { Iris danfordiae (Baker) } \\
\text { Boiss. }\end{array}$} & $\begin{array}{l}\text { Graz - nursery } \\
\text { (purchased) } 1986\end{array}$ & 2000 & Flower bed & \\
\hline & $\begin{array}{l}\text { Zagreb - nursery (pur- } \\
\text { chased) } 2000\end{array}$ & 2017 & Flower bed & \\
\hline Iris decora Wall. (cult.) & Halle 1997 (seed) & 1998 & Nursery & $\begin{array}{l}\text { arrived as "I. sub- } \\
\text { genus Nepalensis" }\end{array}$ \\
\hline $\begin{array}{l}\text { Iris ensata Thunb. } \\
\text { 'Kalići's Ghost' }\end{array}$ & $\begin{array}{l}\text { Gift ("Kalići" Nursery, } \\
\text { Vodnjan) } 2018\end{array}$ & 2019 & Flower bed & $\begin{array}{l}\text { (unregistered } \\
\text { cultivar) }\end{array}$ \\
\hline Iris $\times$ hollandica & $\begin{array}{l}\text { Zagreb - nursery (pur- } \\
\text { chased) } 2017\end{array}$ & 2019 & Flower bed & $\begin{array}{l}\text { I. } \times \text { hollandica } \\
\text { group: I. tingitana } \\
\times \text { I. xiphium }\end{array}$ \\
\hline $\begin{array}{l}\text { Iris } \times \text { norrisii (L.W.Lenz) } \\
\text { C.Whitehouse }\end{array}$ & Utrecht 1993 (seed) & 2001 & Flower bed & $\begin{array}{l}\times \text { Pardancanda nor- } \\
\text { risii L.W.Lenz }\end{array}$ \\
\hline \multirow[t]{2}{*}{$\begin{array}{l}\text { Iris pallida Lam. } \\
\text { 'Variegata' }\end{array}$} & Gift (nn) 1963 & 1969 & Nursery & \\
\hline & Gift (Beograd) 2017 & 2019 & Iridarium & \\
\hline $\begin{array}{l}\text { Iris pseudopallida L. } \\
\text { 'Variegata' }\end{array}$ & Gift (dr. Regula) 2019 & 2019 & Nursery & \\
\hline \multirow[t]{4}{*}{ Iris reticulata M. Bieb. } & Gift (nn) 1963 & 2000 & Flower bed & \multirow{4}{*}{$\begin{array}{l}\text { "horticult. var.?", } \\
\text { "cv.", "Hyb." } \\
\text { Various forms and } \\
\text { colours }\end{array}$} \\
\hline & Sopron (purchased) 1985 & 1985 & Flower bed & \\
\hline & $\begin{array}{l}\text { Zagreb - nursery } \\
\text { (purchased) } 2004\end{array}$ & 2005 & Alpinum & \\
\hline & $\begin{array}{l}\text { Zagreb - nursery } \\
\text { (purchased), } 2000\end{array}$ & 2005 & Flower beds & \\
\hline \multirow[t]{2}{*}{$\begin{array}{l}\text { Iris sanguinea Donn ex } \\
\text { Hornem. 'Snow Queen' }\end{array}$} & Porrentruy 1989 (seed) & 2019 & Iridarium & \\
\hline & Sofia 1990 (seed) & 1993 & Nursery & \\
\hline \multirow[t]{2}{*}{$\begin{array}{l}\text { Iris sibirica L. 'Phosphor- } \\
\text { flamme' }\end{array}$} & Riga 1990 (seed) & 2019 & Flower beds & incorrect \\
\hline & Riga 2019 & 2019 & Flower beds & correct \\
\hline $\begin{array}{l}\text { Iris sibirica L. 'White } \\
\text { Swirl' }\end{array}$ & $\begin{array}{l}\text { Zagreb - nursery (pur- } \\
\text { chased), } 2000\end{array}$ & 2006 & Flower beds & \\
\hline $\begin{array}{l}\text { Iris versicolor L 'Mint } \\
\text { Fresh' }\end{array}$ & Przemysl 2006 (seed) & 2019 & Flower beds & \\
\hline $\begin{array}{l}\text { Iris versicolor L. 'Be- } \\
\text { tween the Lines' }\end{array}$ & Przemysl 2007 (seed) & 2019 & Flower beds & \\
\hline $\begin{array}{l}\text { Iris versicolor L. 'Ker- } \\
\text { mesina' }\end{array}$ & Poznan 1995 (seed) & 2004 & Flower beds & incorrect \\
\hline $\begin{array}{l}\text { Iris versicolor L. 'Party } \\
\text { Line' }^{\prime}\end{array}$ & Przemysl 2006 (seed) & 2019 & Flower beds & \\
\hline
\end{tabular}

*if not stated differently, the plants are obtained in the form of bare roots/rhizomes/bulbs. 


\section{Barbata Elatior ("tall bearded") group of cultivars with complex ancestry}

Since the late 1990s, the largest part of our Iris collection has been composed of hybrids, mostly from the Barbata Elatior or the "tall bearded" (I. × germanicaand I. variegata- hybrids) group. These are regularly obtained in the form of "bare rhizomes", while their exquisite flower characteristics (brightly coloured and enlarged tepals: upper, called "the standards", and lower called "the falls") could not be achieved from seeds. The oldest still living tall bearded irises that we grow today arrived in 1964 from the famous Czech collection near Prague (Průhonice Park and Chateau with the Institute of Botany), as gifts from the former curator Dr. Milan Blažek to our late Garden Manager Dr. Sala Ungar (1908-1988). These are the tall bearded hybrids Iris 'Gaylord', 'Girl Friday', 'Cloud Cap', 'Jane Phillips', 'Goldfackel', 'Olympic Star', 'Pink Plume' and 'Sable Night'. However, most of our recent bearded hybrids were sent during the 1990s by the former Canadian Royal Botanical Gardens (Hamilton, Ontario) curator of Croatian origin, Mr. Charles D. Holetich, to our (now retired) Garden Manager Dr. Ljerka Regula, a vigorous Iris collector herself. Our bearded irises are recently displayed to the public mostly in our little Garden Iridarium, where we have arranged them according to their flower patterns (PFEIFFER, 2015; http:// wiki.irises.org/Main/TallBearded). Some additional data on the bearded irises flower patterns are added as the supplement to Photo-table 4, which depicts the "colours" in our collection.

Tab. 4. Iris (Barbata, Bearded) hybrids, grown from the rhizomes in the Botanical Garden from 1964 to 2019

\begin{tabular}{|c|c|c|c|c|}
\hline Iris cultivar/hybrid & $\begin{array}{l}\text { Origin and year } \\
\text { of obtaining }\end{array}$ & $\begin{array}{l}\text { Last } \\
\text { recorded }\end{array}$ & $\begin{array}{l}\text { Garden } \\
\text { Collection }\end{array}$ & Notes and remarks \\
\hline \multicolumn{4}{|c|}{ Barbata Elatior (Tall Bearded) group } & \\
\hline Acrobat & Hamilton 1998 & 2019 & Nursery & \\
\hline Afternoon Delight & Hamilton 2003 & 2011 & & \\
\hline Autumn Leaves & Gift (Mr. Golob) & 2019 & Nursery & \\
\hline Back in Black & Hamilton 2003 & 2004 & & \\
\hline \multirow[t]{2}{*}{ Banjo Man } & Hamilton 2002 & 2019 & Iridarium & Stolen in 2004,1 plant left \\
\hline & Kalići 2019 & 2019 & Nursery & \\
\hline Before the Storm & Hamilton 1998 & 2019 & Iridarium & \\
\hline Black Prince & Kalići 2019 & 2019 & Nursery & \\
\hline Black Taffeta & Hamilton 1997 & 1998 & & \\
\hline Blue Ensign & Průhonice 1964 & 2019 & Iridarium & not entirely to description \\
\hline \multirow[t]{2}{*}{ Blue Sapphire } & Hamilton 1995 & 2019 & Iridarium & "mixed with 'Eleanor's Pride"”' \\
\hline & Průhonice 1965 & 2004 & & \\
\hline Blue Shimmer & Průhonice 1964 & 1964 & & \\
\hline Blue Staccato & Hamilton 1998 & 2019 & Iridarium & \\
\hline Bold Encounter & Liepāja 2019 & 2019 & Nursery & \\
\hline
\end{tabular}




\begin{tabular}{|c|c|c|c|c|}
\hline Iris cultivar/hybrid & $\begin{array}{l}\text { Origin and year } \\
\text { of obtaining }\end{array}$ & $\begin{array}{l}\text { Last } \\
\text { recorded }\end{array}$ & $\begin{array}{l}\text { Garden } \\
\text { Collection }\end{array}$ & Notes and remarks \\
\hline \multicolumn{4}{|c|}{ Barbata Elatior (Tall Bearded) group } & \\
\hline Bolero & Riga 2019 & 2019 & Nursery & $\begin{array}{l}\text { Not registered. Hybridizer: } \\
\text { Laimonis Zakis }\end{array}$ \\
\hline Brindled Beauty & Cayeux 2019 & 2019 & Nursery & \\
\hline Bronze Bell & Hamilton 1995 & 2004 & & \\
\hline Brussels & Liepāja 2019 & 2019 & Nursery & \\
\hline Buduārs & Riga 2019 & 2019 & Nursery & $\begin{array}{l}\text { Not registered. Hybridizer: } \\
\text { Laimonis Zakis }\end{array}$ \\
\hline Butterscotch Kiss & Hamilton 1999 & 2019 & Iridarium & \\
\hline By Night & Hamilton 2003 & 2003 & & \\
\hline Camelot Rose & Hamilton 1995 & 2019 & Iridarium & \\
\hline Canadian Northland & Hamilton 1995 & 2019 & Flower bed & $\begin{array}{l}\begin{array}{l}\text { Not registered. Hybridizer: } \\
\text { unknown }\end{array} \\
\end{array}$ \\
\hline Cantina & Kalići 2019 & 2019 & Nursery & \\
\hline Caroline (?) & Hamilton 1997 & 2019 & Iridarium & Probably 'Caroline Jane' \\
\hline Cascadian & Průhonice 1964 & 1964 & & \\
\hline Cayenne Capers & Hamilton 2001 & 2004 & & Stolen \\
\hline Changing Seasons & Cayeux 2019 & 2019 & Nursery & \\
\hline Chippendale & Hamilton 1998 & 2019 & Iridarium & \\
\hline Cloud Cap & Průhonice 1964 & 2019 & Iridarium & "mixed with 'Pink Plume' " (?) \\
\hline Collage & Hamilton 2002 & 2002 & & Stolen \\
\hline Colour Splash & Hamilton 1998 & 2019 & Iridarium & not entirely to description \\
\hline Congratulations & Hamilton 2002 & 2019 & Iridarium & Stolen in 2004, 1 plant left \\
\hline Conjuration & Hamilton 2001 & 2002 & & \\
\hline Crinoline & Hamilton 2003 & 2019 & Iridarium & \\
\hline Dangerous Mood & Cayeux 2019 & 2019 & Nursery & \\
\hline Dark Freeze & Kalići 2019 & 2019 & Nursery & \\
\hline Decolletage & Hamilton 2003 & 2019 & Iridarium & Stolen in 2004,1 plant left \\
\hline Deft Touch & Hamilton 1998 & 2019 & Iridarium & \\
\hline Depute Nomblot & Hamilton 1995 & 2000 & & \\
\hline Double Ringer & Liepāja 2019 & 2019 & Nursery & \\
\hline Dūmu Roze & Riga 2019 & 2019 & Nursery & $\begin{array}{l}\text { Not registered. Hybridizer: } \\
\text { Laimonis Zakis }\end{array}$ \\
\hline Dusky Challenger & Hamilton 1995 & 2019 & Flower bed & \\
\hline Eagle's Flight & Hamilton 2003 & 2019 & Iridarium & Stolen in 2004, 2 plants left \\
\hline Ecstatic Night & Hamilton 2001 & 2001 & & \\
\hline Eden & Hamilton 1998 & 2019 & Iridarium & (did not flower for years) \\
\hline El Grandee & Hamilton 1998 & 1999 & & \\
\hline \multirow[t]{2}{*}{ Eleanor's Pride } & Hamilton 1995 & 2019 & Iridarium & mixed with 'Jane Phillips' \\
\hline & Průhonice 1969 & 2004 & & \\
\hline Elegant Girl & Gift (Mr. Golob) & 2019 & Nursery & \\
\hline Elizabeth Noble & Průhonice 1964 & 1975 & & \\
\hline Elzee & Hamilton 1998 & 2004 & & \\
\hline
\end{tabular}




\begin{tabular}{|c|c|c|c|c|}
\hline Iris cultivar/hybrid & $\begin{array}{l}\text { Origin and year of } \\
\text { obtaining }\end{array}$ & $\begin{array}{l}\text { Last } \\
\text { recorded }\end{array}$ & $\begin{array}{l}\text { Garden } \\
\text { Collection }\end{array}$ & Notes and remarks \\
\hline \multicolumn{4}{|c|}{ Barbata Elatior (Tall Bearded) group } & \\
\hline Ethernity & Liepāja 2019 & 2019 & Nursery & $\begin{array}{l}\text { Not registered. Hybridizer: } \\
\text { Laimonis Zakis }\end{array}$ \\
\hline Fabuleux & Cayeux 2019 & 2019 & Nursery & \\
\hline Far Corners & Hamilton 1998 & 2019 & Iridarium & \\
\hline Festive Spirit & Hamilton 1997 & 2019 & Iridarium & \\
\hline Fireball & Hamilton 1995 & 2019 & Iridarium & \\
\hline Firewater & Hamilton 2001 & 2001 & & \\
\hline Flaming Heart & Hamilton 2001 & 2001 & & \\
\hline Fort Apache & Hamilton 1998 & 2001 & & \\
\hline \multirow[t]{2}{*}{ Foxfire } & Hamilton 1995 & 1996 & & \\
\hline & Hamilton 1998 & 2019 & Iridarium & \\
\hline Fringe of Gold & Hamilton 1999 & 2019 & Flower bed & \\
\hline Fuji's Mantle & Hamilton 2002 & 2019 & Iridarium & \\
\hline Galilee & Hamilton 1995 & 2019 & Flower bed & \\
\hline Gay Head & Průhonice 1964 & 1996 & & \\
\hline Gaylord & Průhonice 1964 & 2019 & Iridarium & \\
\hline Gentle Rain & Hamilton 1998 & 2019 & Iridarium & \\
\hline Geometrics & Hamilton 1998 & 2004 & & \\
\hline Girl Friday & Průhonice 1964 & 2019 & Iridarium & not entirely to description \\
\hline Gnu Again & Kalići 2019 & 2019 & Nursery & \\
\hline Going My Way & Hamilton 2001 & 2019 & Iridarium & \\
\hline Goldfackel & Průhonice 1964 & 2019 & Iridarium & \\
\hline Gracie Pfost & Hamilton 2001 & 2001 & & \\
\hline Happy Birthday & Hamilton 2001 & 2019 & Iridarium & \\
\hline Helen McGregor & Průhonice 1964 & 1964 & & \\
\hline Henna Accent & Hamilton 1997 & 2019 & Nursery & incorrect \\
\hline \multirow[t]{2}{*}{ High Command } & Kalići 2019 & 2019 & Nursery & \\
\hline & Průhonice 1969 & 2004 & & Stolen \\
\hline Horny Lorri & Hamilton 2003 & 2019 & Iridarium & incorrect (without 'horns') \\
\hline Ice Cream Castle & Hamilton 1999 & 2019 & Iridarium & \\
\hline Inca Chief & Hamilton 1995 & 2004 & & \\
\hline Incantation & Gift (dr. Regula) & 2019 & Iridarium & \\
\hline Jane Phillips & Průhonice 1964 & 2019 & Iridarium & \\
\hline Jewel Tone & Hamilton 1995 & 2019 & Iridarium & \\
\hline Kona Coast & Hamilton 1998 & 2019 & Iridarium & \\
\hline Laced Cotton & Hamilton 2003 & 2003 & & \\
\hline \multirow[t]{2}{*}{ Ladyfriend } & Hamilton 1998 & 2019 & Iridarium & \\
\hline & Gift (Mr. Golob) & 2019 & Nursery & \\
\hline
\end{tabular}




\begin{tabular}{|c|c|c|c|c|}
\hline Iris cultivar/hybrid & $\begin{array}{l}\text { Origin and year of } \\
\text { obtaining }\end{array}$ & $\begin{array}{l}\text { Last } \\
\text { recorded }\end{array}$ & $\begin{array}{l}\text { Garden } \\
\text { Collection }\end{array}$ & Notes and remarks \\
\hline \multicolumn{4}{|c|}{ Barbata Elatior (Tall Bearded) group } & \\
\hline Laurie & Hamilton 2002 & 2019 & Iridarium & \\
\hline Lights On & Průhonice 1964 & 2019 & Nursery & \\
\hline Liseré Pourpre & Liepāja 2019 & 2019 & Nursery & \\
\hline Loop the Loop & Hamilton 1997 & 2001 & & \\
\hline Lord Dandy & Liepāja 2019 & 2019 & Iridarium & $\begin{array}{l}\text { Not registered. Hybridizer: } \\
\text { Laimonis Zakis }\end{array}$ \\
\hline Lorilee & Hamilton 2003 & 2019 & Iridarium & not entirely to description \\
\hline Louis D'Or & Gift (Mr. Golob) & 2019 & Nursery & \\
\hline Louise Watts & Hamilton 1999 & 1999 & & \\
\hline Lynn Hall & Hamilton 1997 & 2019 & Iridarium & \\
\hline Madeira Belle & Hamilton 1999 & 2004 & & \\
\hline Magic Man & Hamilton 1998 & 2010 & & \\
\hline Majska Andromeda & Gift (Mr. Golob) & 2019 & Nursery & \\
\hline Majska Galaksija & Gift (Mr. Golob) & 2019 & Nursery & \\
\hline Majska Katleja & Gift (Mr. Golob) & 2019 & Nursery & \\
\hline Majska Solistka & Gift (Mr. Golob) & 2019 & Nursery & \\
\hline Majski Brokat & Gift (Mr. Golob) & 2019 & Nursery & \\
\hline Malaysia & Hamilton 1998 & 2004 & & \\
\hline Maori King & Hamilton 1995 & 2004 & & Stolen \\
\hline Margarita & Hamilton 1997 & 2000 & & \\
\hline Memphis Delight & Hamilton 1998 & 1999 & & \\
\hline Michele Taylor & Hamilton 2003 & 2004 & & Stolen \\
\hline Minisa & Hamilton 1997 & 2004 & & \\
\hline Mirror Image & Hamilton 1997 & 1999 & & Stolen \\
\hline Mod Mode & Hamilton 1998 & 2019 & Iridarium & \\
\hline Modra Obzorja & Gift (Mr. Golob) & 2019 & Nursery & \\
\hline Olympic Star & Průhonice 1964 & 2019 & Iridarium & \\
\hline Orange Star & Hamilton 1995 & 1996 & & \\
\hline Orchid Brocade & Hamilton 2003 & 2004 & & Stolen \\
\hline Orelio & Průhonice 1964 & 1964 & & \\
\hline Pagan & Hamilton 1999 & 2019 & Iridarium & \\
\hline Pagan Princess & Průhonice 1964 & 2019 & Iridarium & incorrect \\
\hline Persian Berry & Hamilton 1999 & 2019 & Iridarium & \\
\hline Pink Plume & Průhonice 1964 & 2019 & Iridarium & \\
\hline Pretty Carol & Hamilton 1999 & 1999 & & \\
\hline Prototype & Kalići 2019 & 2019 & Nursery & \\
\hline Queen in Calico & Hamilton 1999 & 2019 & Iridarium & \\
\hline Quetta & Hamilton 1998 & 2019 & Iridarium & \\
\hline
\end{tabular}




\begin{tabular}{|c|c|c|c|c|}
\hline Iris cultivar/hybrid & $\begin{array}{l}\text { Origin and year of } \\
\text { obtaining }\end{array}$ & $\begin{array}{l}\text { Last } \\
\text { recorded }\end{array}$ & $\begin{array}{l}\text { Garden } \\
\text { Collection }\end{array}$ & Notes and remarks \\
\hline \multicolumn{4}{|c|}{ Barbata Elatior (Tall Bearded) group } & \\
\hline Rameses (?) & Bratislava 1983 & 2019 & Iridarium & arrived as 'Schneegoettin' \\
\hline Rapture in Blue & Hamilton 1999 & 2019 & Iridarium & \\
\hline Romantic Melody & Kalići 2019 & 2019 & Nursery & \\
\hline Roy Elliot & $\begin{array}{l}\text { East Grimstead } \\
2000\end{array}$ & 2000 & & Unregistered \\
\hline Royal Regency & Hamilton 1999 & 2019 & Flower bed & \\
\hline Royal Sovereign & Průhonice 1969 & 2019 & Iridarium & \\
\hline Rustic Cedar & Hamilton 1998 & 2004 & & \\
\hline Sable Night & Průhonice 1964 & 2019 & Iridarium & \\
\hline Sable Robe & Hamilton 1997 & 1997 & & \\
\hline Showcase & Hamilton 1998 & 2019 & Iridarium & \\
\hline Silent Majesty & Hamilton 1999 & 2019 & Flower bed & \\
\hline Skylab & Hamilton 1998 & 2004 & & \\
\hline Soaring Kite & Gift (dr. Regula) & 2019 & Iridarium & \\
\hline Sorceress & Hamilton 2001 & 2001 & & Stolen \\
\hline Space Odyssey & Hamilton 1997 & 2019 & Iridarium & \\
\hline Spartan & Hamilton 1998 & 2019 & Iridarium & \\
\hline Spectacular Bid & Gift (dr. Regula) & 2019 & Iridarium & \\
\hline Swazi Princess & Hamilton 1998 & 2019 & Iridarium & \\
\hline Sweet Musette & Hamilton 1999 & 2019 & Iridarium & \\
\hline \multirow[t]{2}{*}{ Tarn Hows } & Hamilton 1995 & 2019 & Iridarium & \\
\hline & Průhonice 1969 & 1996 & & \\
\hline Tea Apron & Hamilton 1999 & 2019 & Iridarium & \\
\hline Tennessee Frost & Hamilton 2002 & 2019 & Iridarium & \\
\hline Theodolinda & Hamilton 2001 & 2019 & Iridarium & \\
\hline Thriller & Hamilton 1998 & 2004 & & \\
\hline Thru Way & Gift (Mr. Golob) & 2019 & Nursery & \\
\hline Tournament Queen & Průhonice 1969 & 2019 & Iridarium & \\
\hline Tranquility & Hamilton 1999 & 2019 & Iridarium & \\
\hline Victoria Falls & Kalići 2019 & 2019 & Nursery & \\
\hline \multirow[t]{2}{*}{ Wabash } & Cayeux 2019 & 2019 & Nursery & \\
\hline & Hamilton 2001 & 2004 & & Stolen \\
\hline Waltzing Princess & Hamilton 1998 & 2019 & Iridarium & \\
\hline White Knight & Kalići 2019 & 2019 & Nursery & \\
\hline Wild Ginger & Hamilton 1999 & 2019 & Iridarium & \\
\hline Wine and Roses & Hamilton 1999 & 2019 & Iridarium & \\
\hline Winner's Circle & Hamilton 2001 & 2019 & Iridarium & \\
\hline Wintry Sky & Kalići 2019 & 2019 & Nursery & \\
\hline
\end{tabular}




\begin{tabular}{|c|c|c|c|c|}
\hline Iris cultivar/hybrid & $\begin{array}{l}\text { Origin and year of } \\
\text { obtaining }\end{array}$ & $\begin{array}{l}\text { Last } \\
\text { recorded }\end{array}$ & $\begin{array}{l}\text { Garden } \\
\text { Collection }\end{array}$ & Notes and remarks \\
\hline \multicolumn{4}{|c|}{ Barbata Elatior (Tall Bearded) group } & \\
\hline Zlata Paleta & Gift (Mr. Golob) & 2019 & Nursery & \\
\hline Zlati Ornat & Gift (Mr. Golob) & 2019 & Nursery & \\
\hline Z-02/YHORN & Gift (Mr. Golob) & 2019 & Nursery & $\begin{array}{l}\text { "yellow, beard extension in the } \\
\text { form of horns - Spaceager". } \\
\text { Hybridizer: Izidor Golob }\end{array}$ \\
\hline Z-03/SAVK & Gift (Mr. Golob) & 2019 & Nursery & $\begin{array}{l}\text { "purple, Spaceager-clone". } \\
\text { Hybridizer: Izidor Golob }\end{array}$ \\
\hline /unknown 1 & Gift (dr. Regula) & 2019 & Iridarium & "two-shades of blue" \\
\hline /unknown 2 & Gift (dr. Regula) & 2019 & Iridarium & "purple dots and dashes" \\
\hline \multicolumn{5}{|c|}{ Barbata Intermedia (Border Bearded) group } \\
\hline Giobe & Kalići 2019 & 2019 & Nursery & \\
\hline Koķete & Riga 2019 & 2019 & Nursery & $\begin{array}{l}\text { Not registered. Hybridizer: } \\
\text { Aleksejs } \\
\text { Muhlinkins }\end{array}$ \\
\hline Making Eyes & Gift (dr. Regula) & 2019 & Nursery & \\
\hline Whoop'em Up & Hamilton 1999 & 2019 & Iridarium & \\
\hline Zelta Dieviņšs & Riga 2019 & 2019 & Nursery & $\begin{array}{l}\text { Not registered. Hybridizer: } \\
\text { Laimonis Zakis }\end{array}$ \\
\hline \multicolumn{4}{|c|}{ Barbata Minima (Miniature Tall Bearded) group } & \\
\hline Consummation & Kalići 2019 & 2019 & Nursery & \\
\hline \multicolumn{4}{|c|}{ Nana (Miniature Dwarf) group } & \\
\hline Piccolo & Gift (prof. Borzan) & 2018 & & Stolen \\
\hline
\end{tabular}

As the results show, since the 1960s, 168 bearded irises have passed through the Garden's Iridarium (Tabs. 4 and 5). Today, we grow 122 cultivars (by far the most from the tall bearded group), among which there are quite many that were recently purchased or received as gifts, to represent the groups of flower patterns that we have been missing in the past (for example, Reverse Amoena, Reverse Bitone, Broken, etc.). The bearded group of irises is - during their time of flowering (late April to early June) - particularly eye-catching to the Garden visitors. This is the reason for this collection having been unfortunately impoverished more than once, mostly due to theft, as can be seen in Tab. 4 .

Tab. 5. Total numbers of Iris taxa grown in Botanical Garden since 1959 and today

\begin{tabular}{|l|l|l|}
\hline Iris collection & Total number (1959-2019) & September 2019 \\
\hline $\begin{array}{l}\text { Indigenous Iris species native to Croatia and } \\
\text { neighbouring countries, brought from the field } \\
\text { excursions as living plants (1959-2019) }\end{array}$ & 24 & 14 \\
\hline $\begin{array}{l}\text { Wild Iris species, not-native to Croatia, grown } \\
\text { from seeds (1955-2019) }\end{array}$ & 66 & 25 \\
\hline $\begin{array}{l}\text { Cultivated varieties of various Iris species } \\
\text { (1960-2019) }\end{array}$ & 15 & 8 \\
\hline $\begin{array}{l}\text { Iris (Barbata,Bearded) hybrids, grown from } \\
\text { rhizomes (1964-2019) }\end{array}$ & 168 & 121 \\
\hline Total & 273 & 168 \\
\hline
\end{tabular}


SOME FINAL REMARKS, FROM THE BOTANIC GARDEN POINT OF VIEW

\section{Wild irises}

Although not in the focus of this paper, several nomenclatural changes within the genus Iris are worth mentioning due to their possible wider consequences.

As a result of DNA sequencing evidence (Goldblatt \& MAbBerley, 2005), a long-known horticultural species Belamcanda chinensis (L.) Leman. was renamed Iris domestica (L.) Goldblatt \& Mabb. Morphologically, and traditionally, this was an unusual decision for the Garden visitors (compared maybe best with the infamous "rearrangement" of the well-known American ornamental asters - Aster novae-angliae and A. novii-belgii - to the unfamiliar and "difficult-to-pronounce" genus Symphyotrichum). It is similar for the former monotypic genus Pardanthopsis (Hance) L.W.Lenz, today again included in the Iris range: Pardanthopsis dichotoma (Pall.) L.W.Lenz (Lenz, 1972) has become Iris dichotoma Pall. (BARKer \& Govaerts, 2016) again. How the nomenclatural complications could be sometimes simplified is illustrated by the case of the the child of the two aforementioned genera: genus $\times$ Pardancanda, a popular example of a genus-hybrid in horticulture $\times$ Pardancanda norrisii L.W.Lenz looks a lot like both of its "parents", Pardanthopsis dichotoma and Belamcanda chinensis - hence the name Pardancanda. But, if the ancestors are actually members of the same genus, then a cross between Iris dichotoma and I. domestica is a much "less spectacular" Iris $\times$ norrisii (L.W.Lenz) C.Whitehouse.

The matter of nomenclature could become even a legal issue, when a genus like Hermodactylus sensu Miller "returns" to the genus Iris Tourn. ex L. (BARKer \& Govaerts, 2016). As already emphasized, all members of the Iris genus in Croatia are statutorily strictly protected in all wild localities - but not Hermodactylus tuberosus (L.) Mill., which is a member of its own genus. Changing the nomenclature, the question arises: does H. tuberosus - now Iris tuberosa L. automatically become statutorily strictly protected, or should it be excluded, as a single non-protected indigenous iris in Croatia? Does it become "all of a sudden" endangered in any way, just by changing its name (several years ago we published in Natura Croatica a paper considering this matter, see Kovačıć et al., 2014)? As with some other "historical" taxa, long-known from Croatian and Balkan flora (for example, Iris croatica, I. illyrica, I. pseudopallida, I. × reichenbachii, I. florentina), today unrecognized by the "western authorities", we shall nevertheless persevere with "Hermodactylus tuberosus", until further notice - or some new re-arrangement of the Iridaceae... After all: "What's in a name? That which we call a rose / By any other name would smell as sweet..."

\section{Cultivated irises}

It is also worth addressing the matter familiar to all botanical garden employees around the globe, which has actually been a problem since the establishment of the first botanical gardens of the modern world in the mid-1550s: thefts, and how to deal with them. 
If there is a common target of "plant lovers who cannot resist", it is a huge group of so called "American" (registered by American Iris Society, AIS) bearded hybrids. These healthy, long-living, and - unfortunately? - very attractive (Phototable 4) plants are exposed to constant threats. Various precautions were taken in our garden and in others during the years (fences, cameras, night-guards...), but except not planting the plants in the open, none of them really worked. As many as 48 of the tall bearded "names" were lost over the years: not all by thefts, but most of those stated as such in Tab. 4 vanished during our last larger rearrangement of the collections in 2004. Our Iridarium is recently under reconstruction again, while we are planning to put a new fence ("cage", could be a better choice of word) around the iris flowerbeds. That, together with "not keeping all eggs in a single basket" is almost all that we can do to preserve these beautiful (and expensive) plants.

A positive thought, for the end: as I said earlier, I have sorted the bearded hybrids into the groups using the original descriptions from their registration data, as much as I could. However, even in our modest collection, some cultivars "got mixed", ending looking quite similar (for example, observe the members of "Variegata"-group in the Photo-table 4). However, if you "have the name" and a plant you are not sure about, it is not so hard these days to identify the existing Tall Bearded iris hybrids using the meticulous descriptions and highquality photos in the Iris-Encyclopaedias and databases. Unfortunately, if you have just a plant, but the name is missing, it is very hard to find an "appropriate" one among almost 50000 registered hybrids! However, the huge social network of iris-lovers is very helpful in finding it: our latest example is the misinterpreted cultivar 'Schneegöttin' (should be pure-white, but the flowers of our plants are multi-coloured), for years without a proper name, concerning which I asked for help from one of such groups. In less than two hours, American "iris-aficionados" detected the hybrid: it is very possibly a 'Ramezes' (Photo-table 4)!

\section{CONCLUSION}

Since the establishment of our Garden, we have grown at least 273 Iris taxa: from the indigenous species and natural varieties collected in their native localities or grown from the seeds, to the cultivars and hybrids obtained by artificial crossings, gained in the forms of rhizomes or bulbs. Our current collection holds 39 wild taxa (14 Croatian native and 25 introduced), and 129 cultivars (eight garden varieties of cultivated taxa and 121 "bearded" hybrids) - a total of 168 . The oldest iris grown in the Garden today is I. × germanica (since 1962), followed by several Barbata Elatior-cultivars that arrived from Průhonice in 1964.

\section{ACKNOWLEDGES}

I wish to thank our retired Garden manager, Dr. Ljerka Regula, for sharing her documentation, personal data and correspondence on the Iris collections during the last 50 years, as well for the tall bearded cultivars which she donated to the 
Garden from her personal collection. The kindness of Dr Milan Blažek (Průhonice, Czech Republic) and Mr Charles D. Holetich (Hamilton, Canada) in sending and bringing tall bearded Iris cultivars in the 1960s and 1990s, respectively, is highly appreciated (Mr. Blažek is still dynamically interested in the plants he donated to the Garden in 1964!). I also want to thank Professor Božena Mitić (Dpt. Of Botany, Faculty of Science, University of Zagreb) for sharing Croatian wild Iris samples from her scientific research; Ms. Alison Benski (Schreiner Iris Gardens, Salem, Oregon, USA) for clarifying many questions on the registration of bearded irises; Inese Nāburga, MSc (Botanical Garden of the University of Latvia, Riga) for kindly replacing our missing cultivar and donating original Latvian tall bearded hybrids; Davor Cetina, MSc ("Kalići" Nursery, Croatia) for happily exchanging and donating plant material, as well as sharing many useful cultivation tips; and renown Iris-hybridizer Mr. Izidor Golob (Kaniža, Slovenia) for donating valuable tall bearded samples of his own invention. Finally, my colleague Dr. Dubravka Sandev, together with our gardeners, helped me very much in sorting and replanting many Iris hybrids and cultivars scattered around the Garden, for which I am most grateful.

Received July 31, 2019

\section{REFERENCES}

American Iris Society. Available from: https://irises.org/ [accessed September 2019].

Barker, C. \& Govaerts, R., 2016: World Checklist of Iridaceae (Iris genus). Facilitated by the Royal Botanic Gardens, Kew. http://apps.kew.org/wcsp/ [accessed July 2019].

CITES (Checklist of CITES Species), 2018. Available from: http://checklist.cites.org/\#/en [accessed July 2019].

EASIN (European Alien Species Information Network), 2012. Available from: http://alien.jrc.ec.europa. eu/SpeciesMapper [accessed July 2019].

Euro+Med (2006-): Euro+Med PlantBase - the information resource for Euro-Mediterranean plant diversity. Published on the Internet http://ww2.bgbm.org/EuroPlusMed/ [accessed July 2019].

Flora Croatica Database - Iris L. Available from: https://hirc.botanic.hr/fcd/ShowResults. aspx?hash=-989949237 [accessed June 2019].

Flora Croatica Database - Red List. Iris. Available from: https://hirc.botanic.hr/fcd/CrvenaKnjiga/ [accessed June 2019].

Goldblatt, P. \& D. J. Mabberley, 2005: Belamcanda included in Iris, and the new combination I. domestica (Iridaceae: Irideae). Novon 15: 128-132.

HeInz, A., 1895-1896: Kr. Botanički vrt u Zagrebu. Glasnik Hrvatskoga naravoslovnoga društva, 8(16), 1-54. (Available from: http://kgzdzb.arhivpro.hr/?kdoc=11012907)

Historic Iris Preservation Society. Available from: https://www.historiciris.org/ [accessed September 2019].

IPNI (The International Plant Names Index), 2018: Available from: https://www.ipni.org/

Iris Encyclopedia of the American Iris Society. Available from: http://wiki.irises.org/Main/TallBearded [accessed July 2019].

IUCN, 2019: The IUCN Red List of Threatened Species. Version 2019-2. https://www.iucnredlist.org

Lenz, L.W., 1972: Pardanthopsis dichotoma (Pallas) L. W. Lenz. El Aliso 7(4), 403.

Kamenetsky, R. \& Окubo, H. (eds.), 2012: Iridaceae. Ornamental Geophytes: From Basic Science to Sustainable Production. CRC Press.

Kovačić, S., 2015: Plethora of plants - Collections of the Botanical Garden, Faculty of Science, University of Zagreb (1): Temperate glasshouse exotics - historic overview. Natura Croatica 24(2), 361-428 $\left(397^{*}\right)$.

Kovačić, S., Sandev, D., Mihelj, D., Stamenković, V., 2014: Win some, lose some - statutorily strictly protected indigenous plant species in the Botanical Garden of the Faculty of Science (University of Zagreb, Croatia). Natura Croatica 23(2), 415-432.

Mitić, B. \& Cigić, P., 2007: Endemic taxa of the Genus Iris L. (Iridaceae) in Croatia. Hrvatska misao 42, 50-81. 
Mitić, B., Nikolić, T. \& Ortynski, N., 2004: Iris croatica Horvat et Horvat M. In: Nikolić, T., Topić, J. (ed.): Red book of vascular flora of Croatia: categories EX, RE, CR, EN and VU. Ministarstvo kulture \& Državni zavod za zaštitu prirode Republike Hrvatske, Zagreb.

National Gardening Organization. Available from: https:/garden.org/plants/view/181474/Irises-Iris/ [accessed September 2019].

Pacific Bulb Society. Available from: https://www.pacificbulbsociety.org/pbswiki/index.php/Iris [accessed July 2019].

Pfeiffer, K., 2015: Iris Flower Patterns. National Gardening Organization, Available from: https:// garden.org/ideas/view/KentPfeiffer/2299/Iris-Flower-Patterns/ )

Pravilnik o strogo zaštićenim vrstama (NN 144/13, 73/16). (Ordinance of strictly protected species). In Croatian. Official Gazette (OG) 73/2016. Available from: http://www.propisi.hr/print.php?id=12728 [accessed June 2019].

The Plant List, 2013: Published online http://www.theplantlist.org/ [accessed June 2019].

Sandev, D., Minelu, D. \& Kovačić, S., 2018: Plethora of plants - Collections of the Botanical Garden, Faculty of Science, University of Zagreb (2): Glasshouse succulents. Natura Croatica 27(2), 407430.

Stamenković, V. \& Kovačić, S. (eds.), 2014: Fifty Sights at the Botanical Garden - for Passers-by, Strollers and Real Enthusiasts. A Guide to the Botanical Garden, Faculty of Science, University of Zagreb. Authors: Juretić, B., Kovačić, S., Mihelj, D., Sandev, D. \& Stamenković, V. Faculty of Science, University of Zagreb, Croatia.

Shear, W., 2002: The Gardener's Iris Book. The Taunton Press, CT, USA.

Topić, J., Nikolić, T., Mitić, B., 2004: Iris sibirica L. subsp. sibirica. In: Nikolić, T., Topić, J. (ed.): Red book of vascular flora of Croatia: categories EX, RE, CR, EN and VU. Ministarstvo kulture \& Državni zavod za zaštitu prirode Republike Hrvatske, Zagreb.

World Flora Online, Published on the Internet http://www.worldfloraonline.org/, 30 Jul 2019 (based on The Plant List, 2013: http://www.theplantlist.org/ ) [accessed June 2019].

\section{SAŽETAK}

Obilje bilja - zbirke Botaničkoga vrta Prirodoslovno-matematičkog fakulteta Sveučilišta u Zagrebu (3): Zbirka perunika (Iris, Iridaceae)

\section{S. Kovačić}

Nakon analiza zbirki domaće flore toplog staklenika (Kovačić, 2015) te kaktusa i drugih mesnatica (SANDEV et al., 2018) na redu je analiza zbirke perunika (rod Iris, porodica Iridaceae) koje su, prema dostupnim podacima, rasle u Botaničkom vrtu PMF-a od osnutka 1889. do danas.

Podatci pokazuju da su kroz vrtne zbirke prošle barem 273 svojte perunika: 24 hrvatske vrste i vrste susjednih zemalja (Bosna i Hercegovina, Srbija, Sjeverna Makedonija) donošene $\mathrm{s}$ terenskih istraživanja (planta viva) i sađene na kamenjare - biljnogeografske vegetacijske skupine (krška, mediteranska i submediteranska) s domaćom florom; 66 ostalih divljih vrsta perunika, uzgajanih iz sjemenki naručivanih putem Index (Delectus) Seminum-mreže razmjene između botaničkih vrtova; 15 ukrasnih kultivara, varijeteta i križanaca poznatih vrsta, dijelom uzgajanih iz sjemenki, a dijelom iz podanaka (rizoma) ili lukovica; te 168 križanaca složenog podrijetla iz skupine tzv. „bradatih“ (Barbata, Bearded) perunika, uzgajanih isključivo iz podanaka.

S krajem rujna 2019. u Botaničkom vrtu živjelo je 168 svojti perunika u nekoliko zbirki (kamenjare s domaćom florom, sistematsko polje, klijališta, ukrasne površine, Iridarij). 


\section{Photo-table 1}

Six indigenous Croatian Iris-species, grown in the phytogeographical rockeries with indigenous flora since the 1960s, brought from their native localities. Iris variegata (bottom right) is not currently in our collection. Details in Table 1. Authors: Mirna Kirin (MK) and Sanja Kovačić (SK).

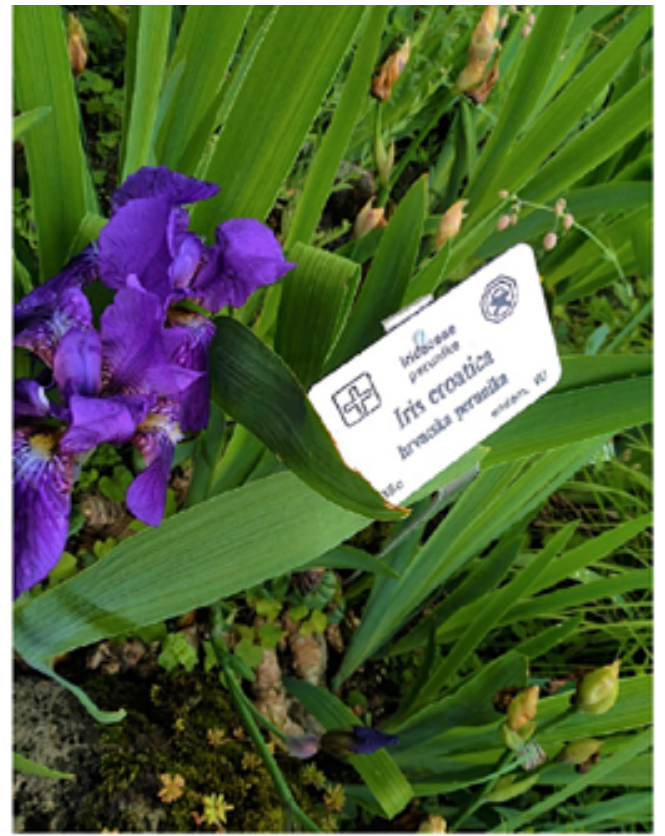

Iris croatica (SK)

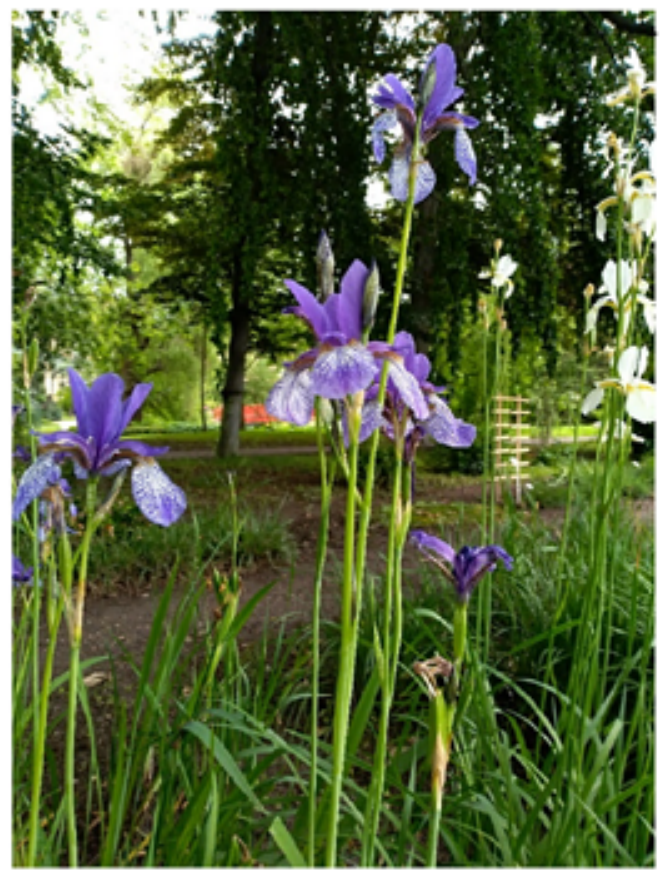

Iris sibirica (SK)
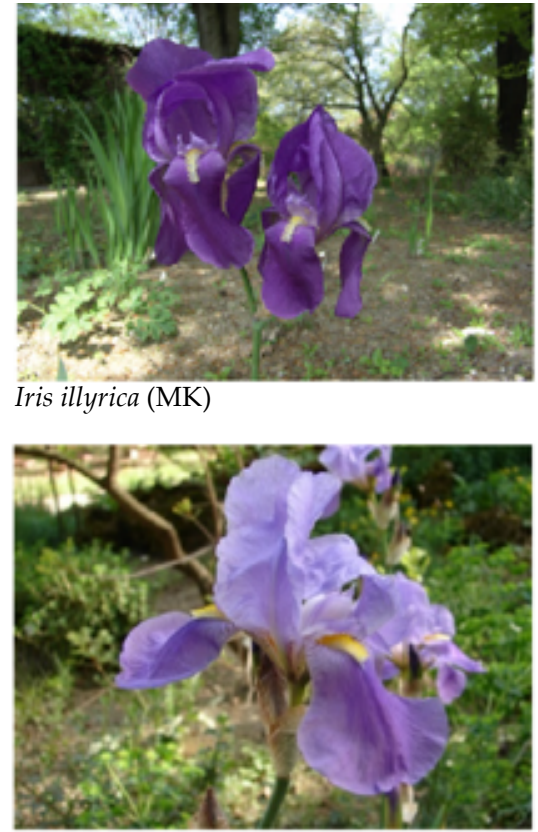

Iris pallida (SK)

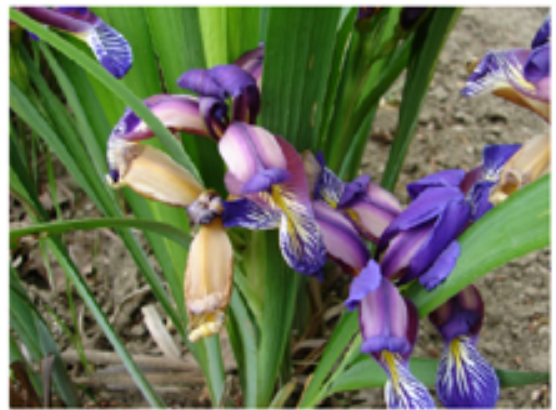

Iris graminea $(\mathrm{MK})$

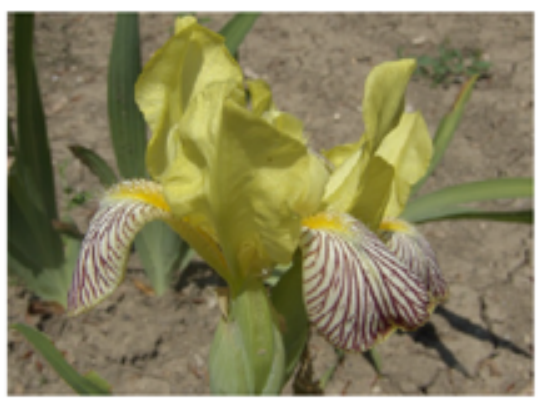

Iris variegata $(\mathrm{MK})$ 


\section{Photo-table 2}

Nine wild Iris-species, grown from seed in the nurseries, systematic fields and flowerbeds. Iris biglumis (middle right) is not currently in our collection. Details in Table 2. Authors: Mirna Kirin (MK) and Sanja Kovačić (SK).

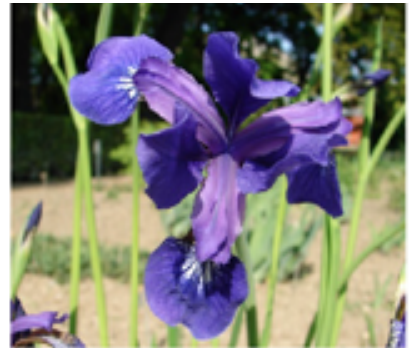

Iris bulleyana (SK)

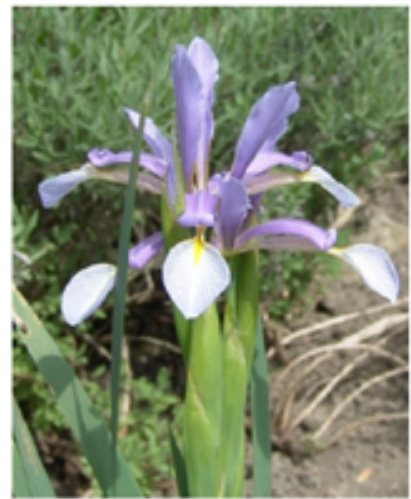

Iris halophila (MK)

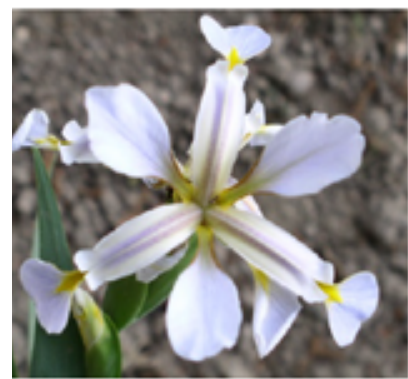

Iris (halophila ssp.) sogdiana (MK)

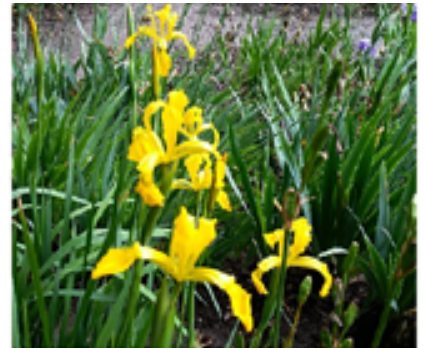

Iris crocea (SK)

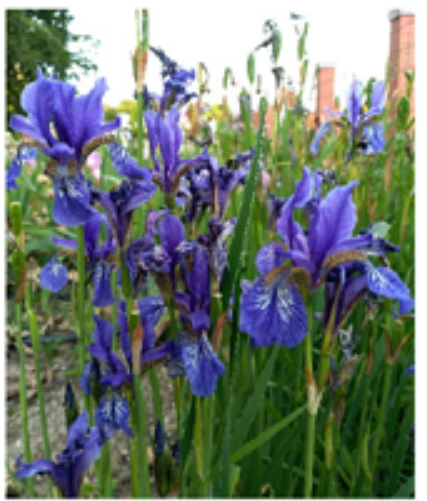

Iris sanguinea (SK)

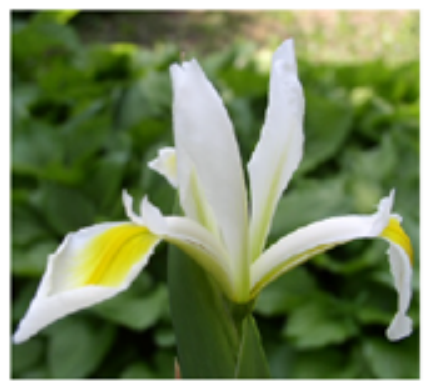

Iris orientalis (I. ochroleuca, MK)

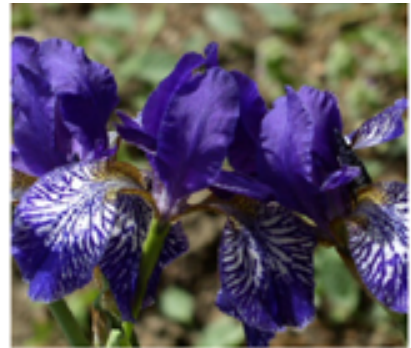

Iris sikkimensis (MK)

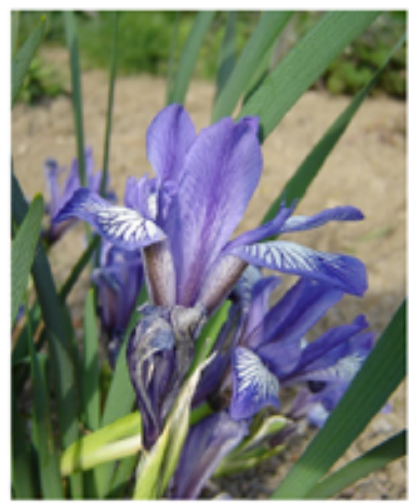

Iris biglumis (MK)

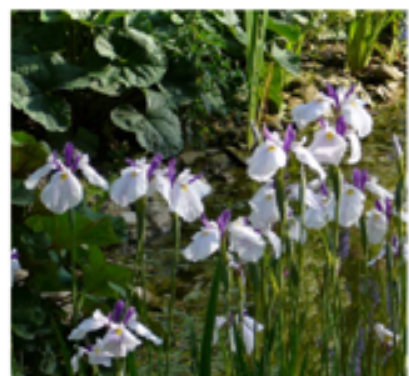

Iris kaempferi (MK) 


\section{Photo-table 3}

Five Iris varieties, cultivars and hybrids of different taxonomic ranks, grown from the seed or obtained as living plants, planted in the nurseries, systematic fields and flowerbeds. Iris sibirica 'White Swirl' (upper right) and cultivars of I. reticulata (bottom left) are not currently in our collections. Iris $\times$ germanica (bottom right), once considered to be indigenous in Croatia, is the oldest iris still living in our collection (since 1962). Details in Table 3. Authors: Mirna Kirin (MK) and Sanja Kovačić (SK).

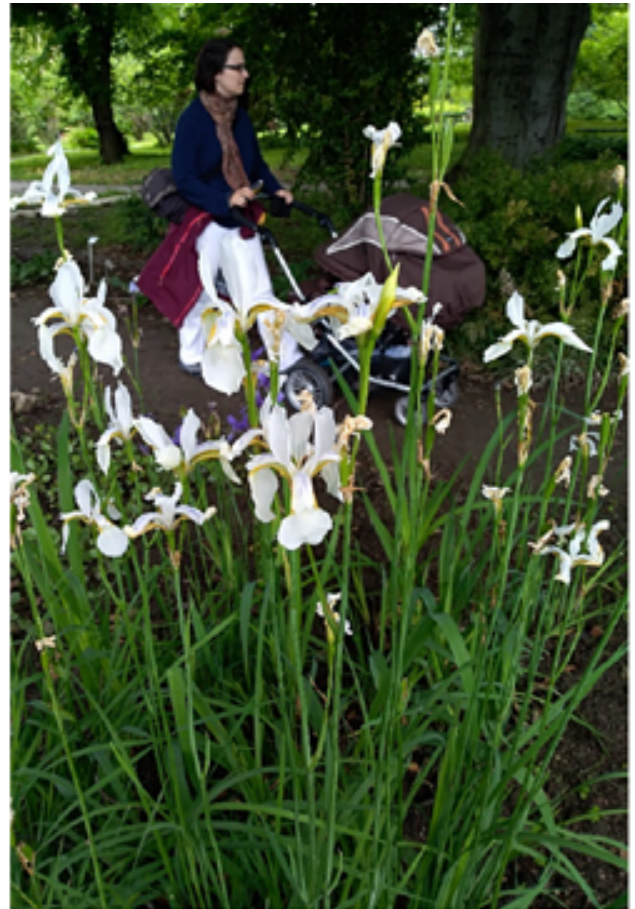

Iris sanguinea 'Snow Queen' (SK)

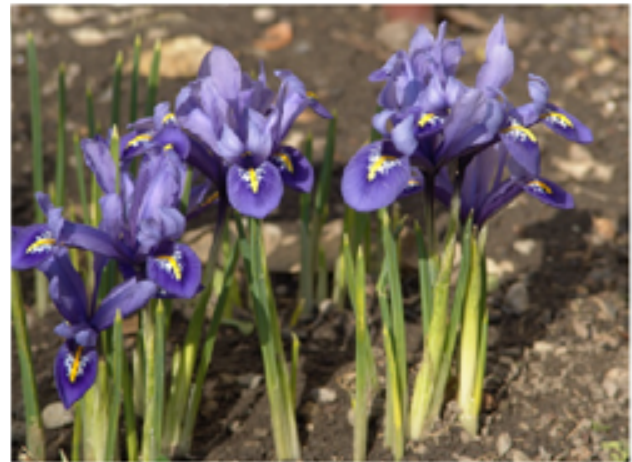

Iris reticulata cult. (MK)

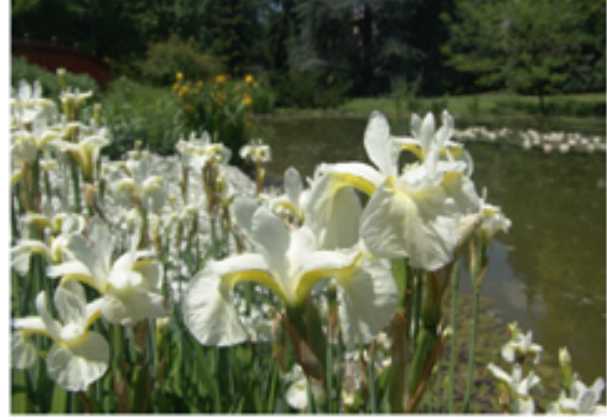

Iris sibirica 'White Swirl' (MK)

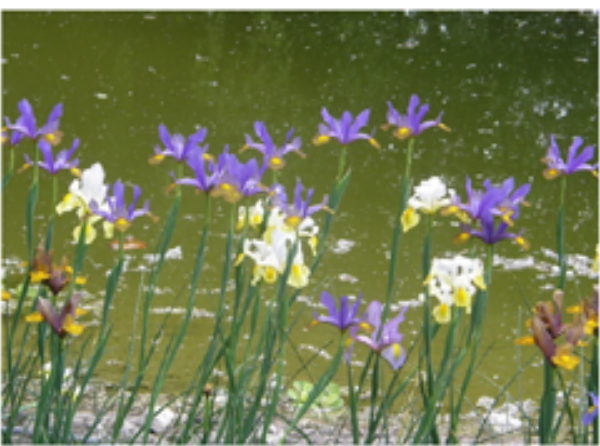

Iris $x$ hollandica cult. (MK)

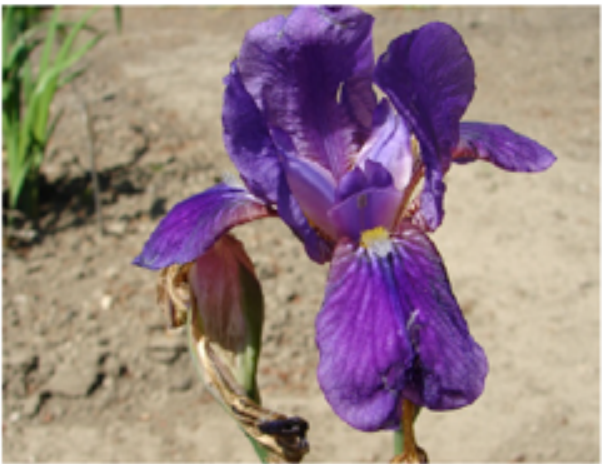

Iris $x$ germanica cult. (MK) 
Photo-table 4 (abcd)

Thirty-five from a total of 168 Iris Barbata (bearded) hybrids grown in our collection, arranged to represent eight large groups of these cultivars, assembled by their flower patterns. A careful observer will detect sometimes very subtle differences among individual groups and hybrids. Details in Table 4. Authors: Mirna Kirin (MK) and Sanja Kovačić (SK).

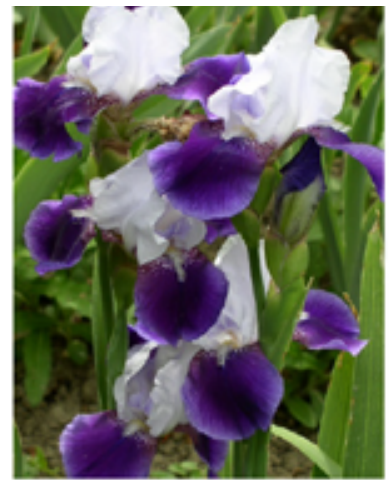

Amoena-group:

Iris BE 'Gaylord' (MK)

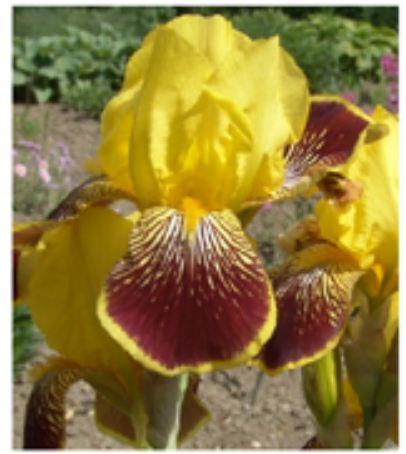

Variegata-group:

Iris BE 'Maori King' (MK)

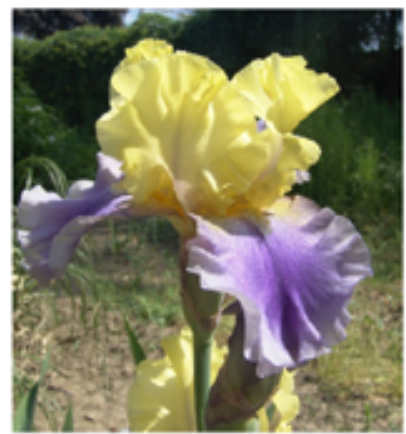

Bicolor-group:

Iris BE 'Edith Wolford' (MK)

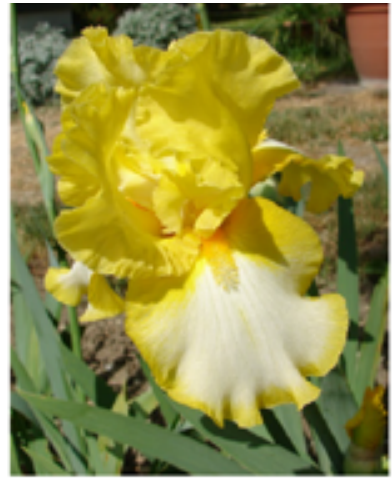

Debbie Rairdon-group:

Iris BE 'Fringe of Gold' (MK)

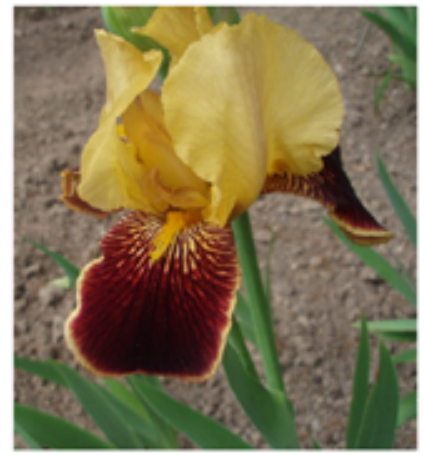

Variegata-group:

Iris BE 'High Command' (MK)

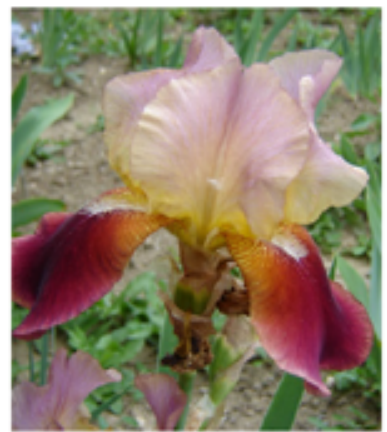

Bicolor-group:

Iris BE 'Olympic Star' (MK)

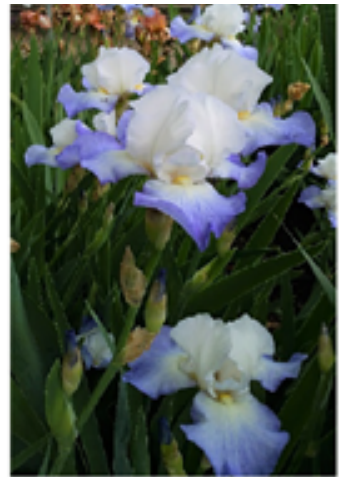

Emma Cook-group:

Iris BE 'Fuji's Mantle' (SK)

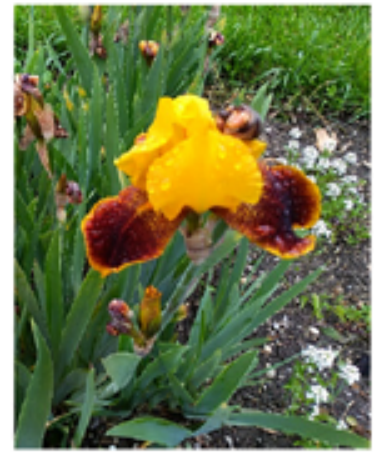

Variegata-group:

Iris BB 'Whoop'em Up' (SK)

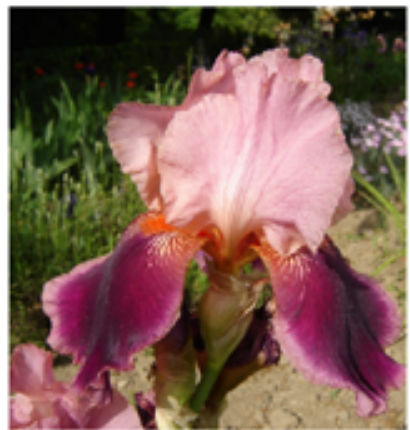

Bicolor-group:

Iris BE 'Wine and Roses' (MK) 


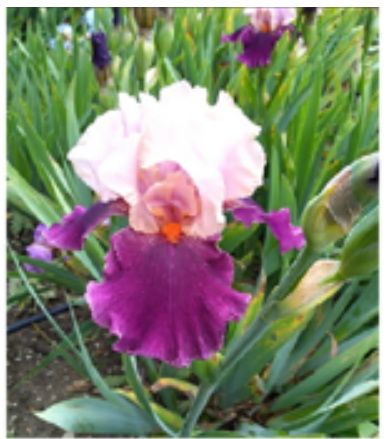

Bicolor-group:

Iris BE 'Color Splash' (SK)

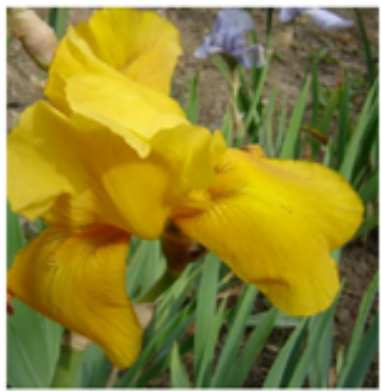

Bitone-group:

Iris BE 'Goldfackel' (SK)

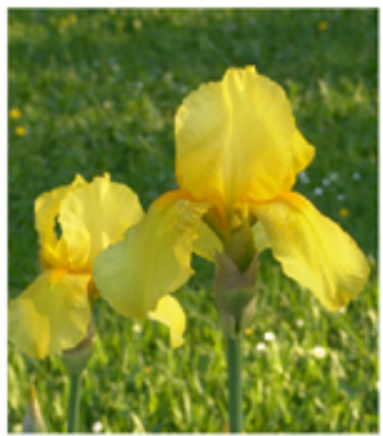

Self-group:

Iris BE 'Foxfire' (MK)

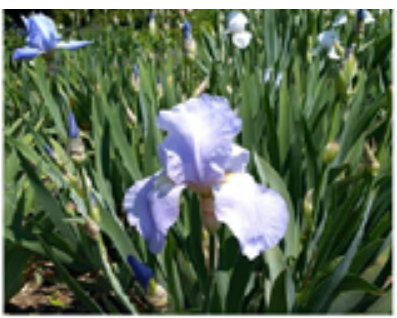

Self-group:

Iris BE ‘Jane Phillips' (SK)

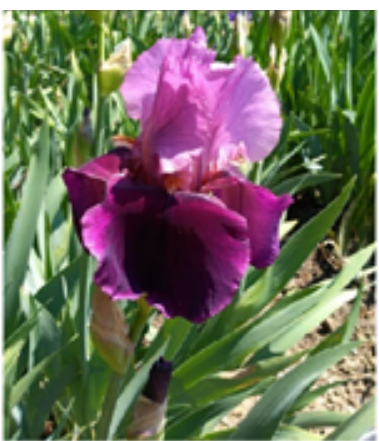

Bitone-group:

Iris BE 'Camelot Rose' (SK)

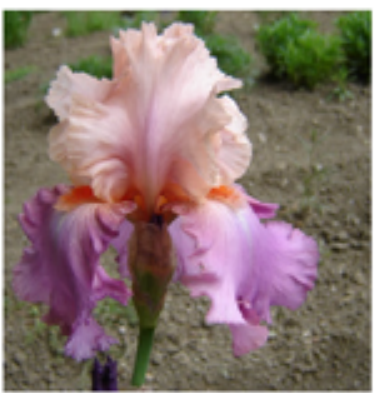

Bitone-group:

Iris BE 'Sweet Musette' (MK)

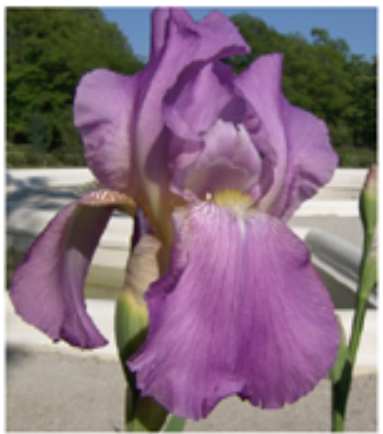

Self-group:

Iris BE 'Pink Plume' (MK)

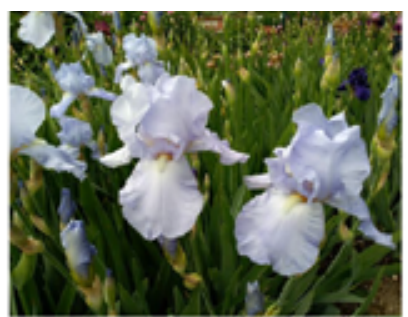

Self-group:

Iris BE 'Eleanor's Pride' (SK)

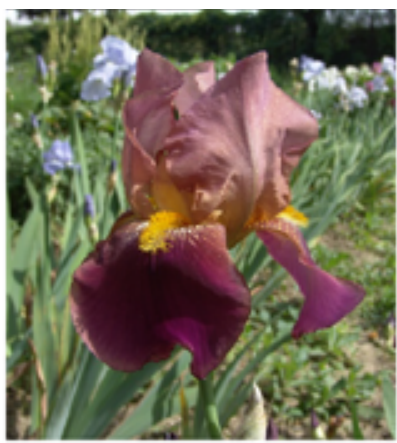

Bitone-group:

Iris BE 'Député Nomblot' (MK)

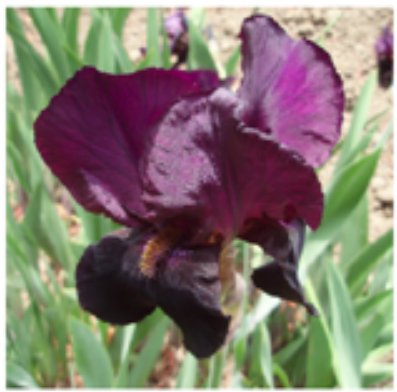

Bitone-group:

Iris BE 'Sable Night' (MK)

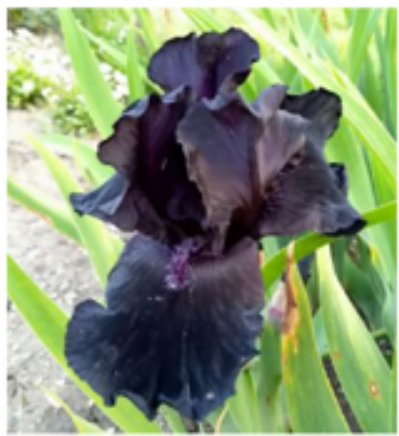

Self-group:

Iris BE 'Before the Storm' (SK)

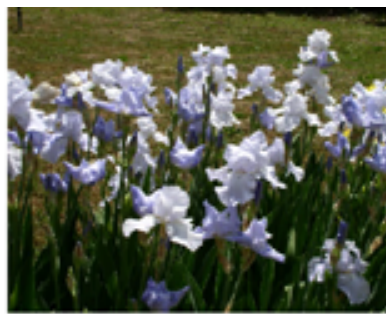

Self-group: Iris BE 'Eleanor's

Pride' - a 'tricolor' clump (MK) 


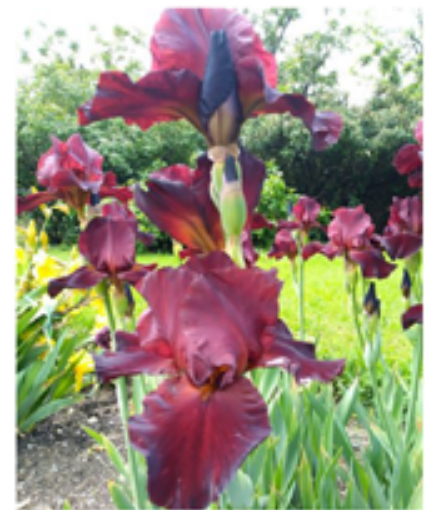

Self-group:

Iris BE 'Spartan' (SK)

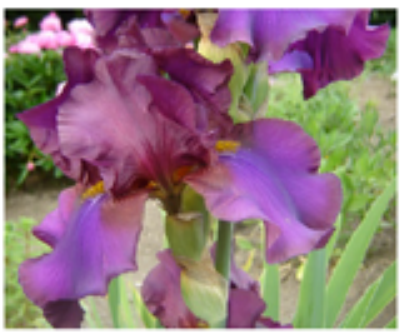

Blend-group:

Iris BE 'Pagan' (MK)

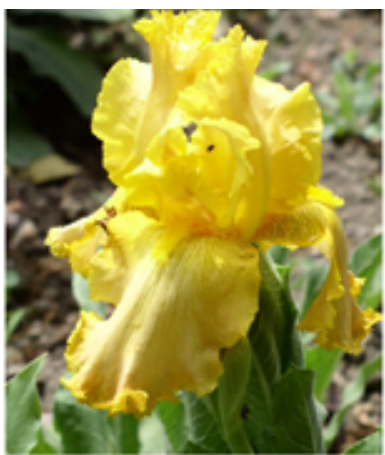

Blend-group:

Iris BE 'Butterscotch Kiss' (MK)

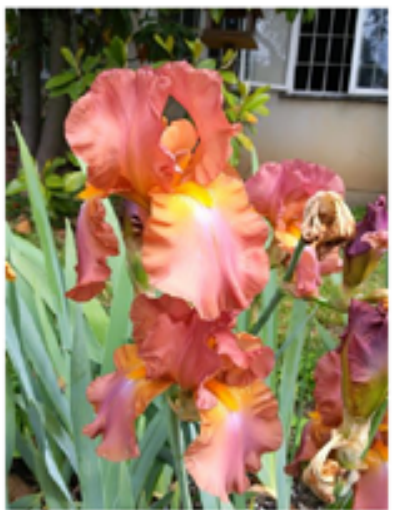

Blend-group:

Iris BE 'Chippendale' (SK)

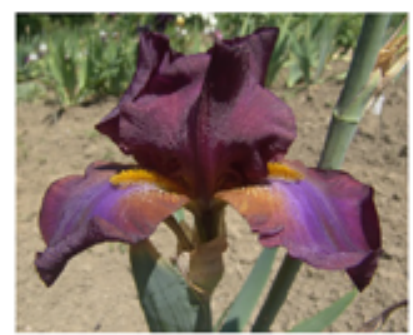

Blend-group:

Iris BE 'Banjo Man' (MK)

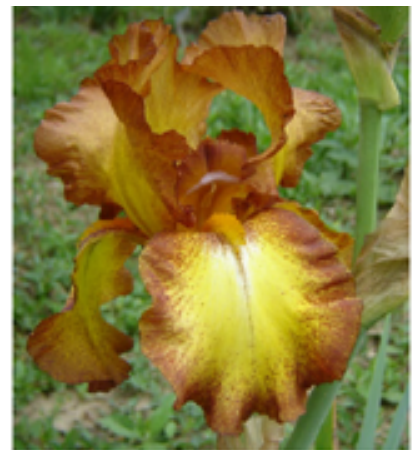

Plicata-group:

Iris BE 'Kona Coast' (MK)

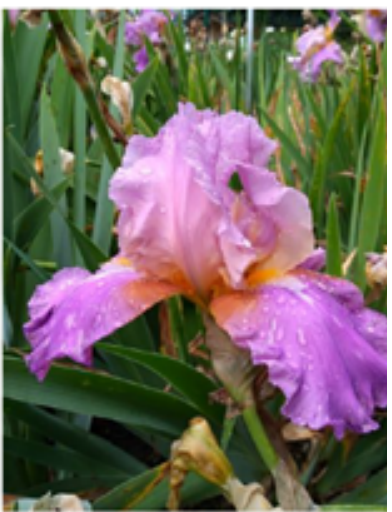

Blend-group:

Iris BE 'Laurie' (SK)

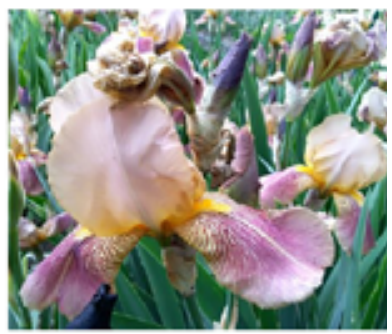

Blend-group:

Iris BE aff. 'Rameses' (SK)

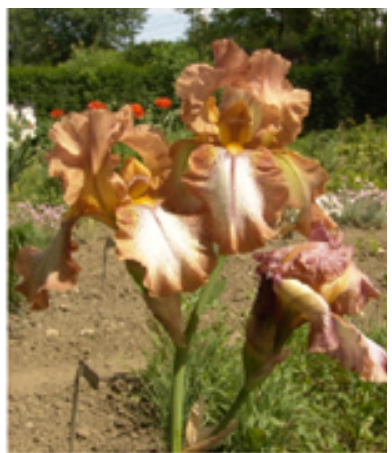

Plicata-group:

Iris BE 'Wild Ginger' (MK)

\section{Photographs:}

All photographs in Photo-tables 1, 2, 3 and 4abcd are originals, taken between 1999 and 2019 in the collections of the Botanical Garden of the Faculty of Science (University of Zagreb) by Mirna Kirin (MK), member of the "Friends of Botanical Garden" group, and Dr Sanja Kovačić (SK), senior Garden curator. 


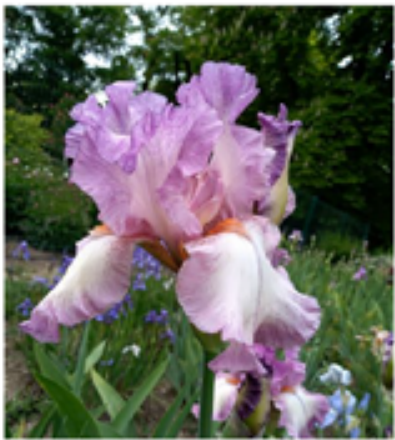

Plicata-group:

Iris BE 'Waltzing Princess' (SK)

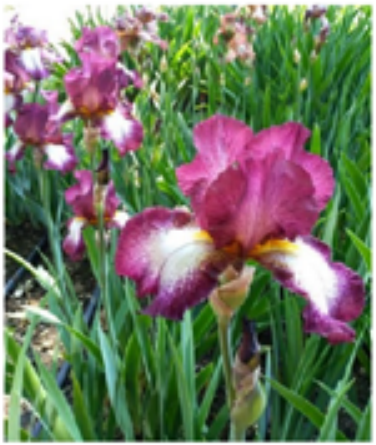

Plicata-group: Iris BE 'Crinoline' (SK)

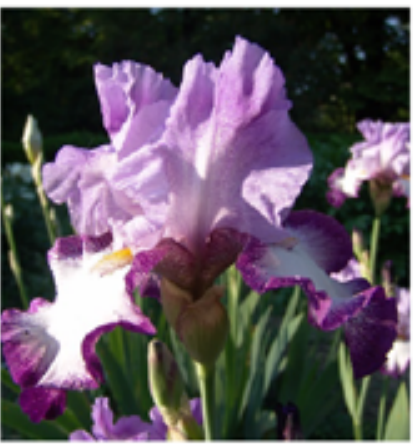

Plicata-group:

Iris BE 'Eagle's Flight' (MK)

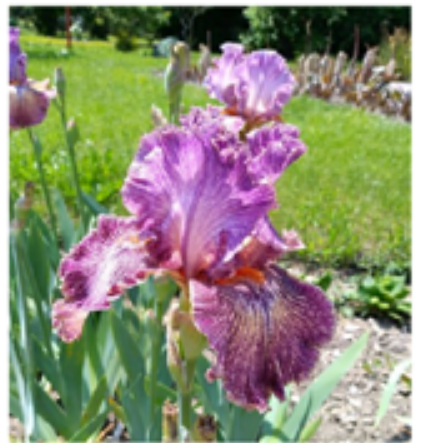

Plicata-group:

Iris BE 'Queen in Calico' (SK)

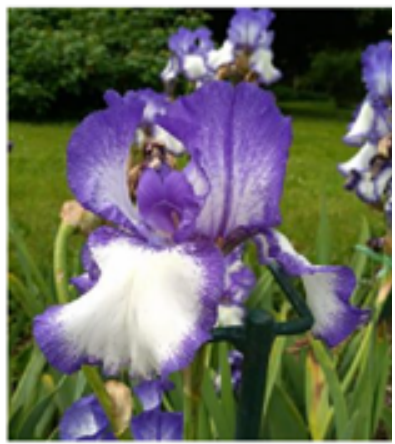

Plicata-group:

Iris BE 'Blue Staccato' (SK)

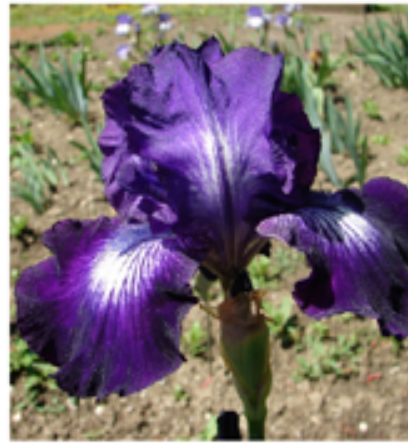

Plicata-group:

Iris BE 'Winner's Circle' (MK)

Short description of the tall bearded Iris-groups depicted in Photo-table 4abcd, assembled according to their flower patterns (PFEIFfer 2015):

"Self" - flower tepals (standards and falls) are in the same colour; "Bitone" - tepals are in different shades (tones) of the same colour, while the falls are darker; "Bicolor" - standards are in different colour from the falls; "Amoena" - specific type of bicolor, where the standards are white and the falls are coloured; "Variegata" - a specific type of bicolor, where the standards are yellow(ish) and the falls are brown(ish) to purple(ish); "Emma Cook" - derived from amoena/bicolor breeding, but with just a band of pigmentation around the border of the falls; "Debbie Rairdon" - coloured standards and white falls with a band of standard colour around the edges; "Blend" - combination of two or more colours which are smoothly or unevenly mixed; "Plicata" - light ground colour with anthocyanin pigment stippled, dotted, or stitched around the margins of tepals.

Kratak opis skupina križanaca bradatih perunika (Barbata, "Bearded") prikazanih u foto-tabli 4abcd, prema uzorcima boja cvjetova (Pfeiffer, 2015):

"Self" - ocvijeće ("standards" = gornji listovi ocvijeća, neformalno također zvani i "latice", te "falls" = donji listovi ocvijeća, "lapovi”) je jednobojno; "Bitone" - ocvijeće je različitih tonova (nijansi) iste boje, s time da su donji listovi tamniji; "Bicolor" - gornji i donji listovi ocvijeća različitih su boja; "Amoena" - skupina dvobojnih kultivara kojima su gornji listovi ocvijeća bijeli, a donji žuti do smeđi; "Variegata" - skupina dvobojnih kultivara kojima su gornji listovi žuti, a donji smeđi do purpurni; "Emma Cook" - skupina križanaca između „amoena“ i „bicolor“ skupina, koje oko donjih listova ocvijeća nose tek tanak prsten u boji gornjih listova; "Debbie Rairdon" - križanci obojenih gornjih listova ocvijeća i bijelih donjih, koji rubom nose tanak prsten u boji gornjih listova; "Blend" - kombinacija dviju ili više boja koje se jednolično ili nejednolično stapaju ili miješaju; "Plicata" - križanci svijetle temeljne boje (bez antocijanina) preko koje je rubom listova ocvijeća nanesen uzorak tamnije (antocijaninske) boje u obliku točkica, crtica ili „šavova“. 

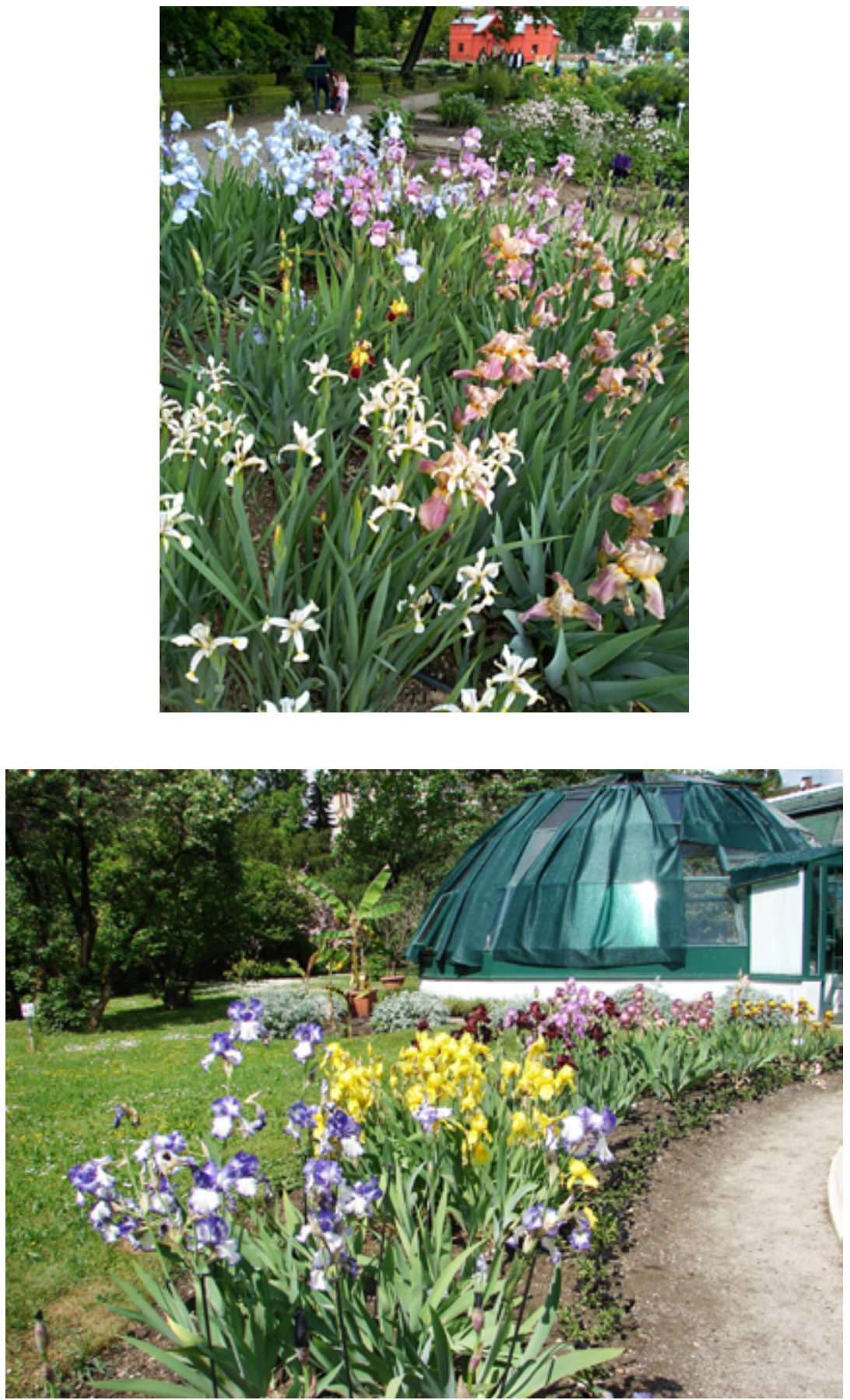

Details from the Iris-collections of the Botanical Garden, Faculty of Science, University of Zagreb. (SK, May 2019) 